\title{
Reconfiguration analysis of a 4-RUU parallel manipulator
}

\author{
Latifah Nurahmi ${ }^{\mathrm{a}} \quad$ Stéphane Caro ${ }^{\mathrm{a} *} \quad{\text { Philippe } \text { Wenger }^{\mathrm{a}}}$ \\ Josef Schadlbauer $^{\mathrm{b}} \quad$ Manfred Husty ${ }^{\mathrm{b}}$ \\ anstitut de Recherche en Communications et Cybernétique de Nantes \\ 1 rue de la Noë, 44321 Nantes, France \\ \{latifah.nurahmi, stephane.caro, philippe.wenger@irccyn.ec-nantes.fr\} \\ ${ }^{\mathrm{b}}$ Unit Geometry and CAD, University of Innsbruck \\ Technikerstraße 13, Innsbruck 6020, Austria \\ \{josef.schadlbauer, manfred.husty@uibk.ac.at\}
}

\begin{abstract}
This paper deals with the characterization of the operation modes of the 4-RUU parallel manipulator with an algebraic approach, namely the Study's kinematic mapping of the Euclidean group $S E(3)$. As the 4-RUU parallel manipulator is an over-constrained manipulator, it can be decomposed into two 2-RUU parallel manipulators. The manipulators are described by a set of constraint equations and the primary decomposition is computed. By combining the results of primary decomposition from two 2-RUU parallel manipulators, it reveals that the 4-RUU parallel manipulator has two Schönflies modes (4-dof) and one lower dimension operation mode (2-dof). The singularity conditions are obtained by deriving the determinant of the Jacobian matrix of the constraint equations with respect to the Study parameters in each operation mode. It is shown that there exist singular configurations in which the manipulators may switch from one operation mode to another operation mode. All the singular configurations are mapped onto the joint space, i.e., the actuated joint angles, and are geometrically interpreted. Eventually, the 4-RUU parallel manipulator may switch from the 1st Schönflies mode to the 2nd Schönflies mode, or vice versa, via the 2-dof third mode that contains self-motions.
\end{abstract}

\footnotetext{
${ }^{*}$ Corresponding author. Email: stephane.caro@irccyn.ec-nantes.fr; Tel: +33 (0)2 40376968
} 


\section{Introduction}

To the best of the authors knowledge, the notion of operation mode was initially introduced by Zlatanov et al. in [1] to explain the behaviour of the three degree-of-freedom (3-dof) DYMO robot which can undergo a variety of transformation when passing through singular configurations. In [2], the author analysed the types of operation modes and the transition configurations of the 3-RER ${ }^{1}$ Parallel Manipulator (PM) based upon the Euler parameter quaternions. Walter et al. in [3] used the Study's kinematic mapping to show that the 3-UPU PM built at the Seoul National University (SNU) has nine different operation modes. Later in [4], the authors revealed five different operation modes of the 3-UPU PM proposed by Tsai in 1996 [5]. By using same approach, Schadlbauer et al. in [6] found two distinct operation modes of the 3-RPS PM proposed by Hunt in 1983 [7]. Later in [8], the authors characterized the motion type in both operation modes by using the axodes. The self-motions of this manipulator were classified in [9]. Another PM of the 3-RPS family is the 3-RPS Cube PM and was proposed by Huang et al. in 1995 [10]. Nurahmi et al. in [11, 12] found that this manipulator has only one operation mode in which the 3-dof general motion and 1-dof Vertical Darboux Motion occur inside the same operation mode.

Accordingly, a general methodology for the type synthesis of reconfigurable mechanisms has been proposed and several new reconfigurable mechanisms have been generated. In [13, 14], the authors proposed a general method based upon the screw theory to synthesize a PM that can perform two operation modes. In [15], a novel 1-dof single-loop reconfigurable 7-R mechanism with multiple operation modes based upon the Sarrus mechanism, was proposed. The following year, the reconfiguration analysis of this mechanism based on the kinematic mapping and the algebraic geometry method was presented in [16].

By using the theory of the displacement groups, the lower-mobility PM with multiple operation modes and different number of dof were presented in [17]. Refaat et al. in [18] introduced a family of 3-dof PM that can exhibit two 1T1R modes by using Lie-group theory. In [19], Gogu introduced several PM with two 2T1R modes. In [20], a new joint was presented and added in the manipulator architecture hence it allows the moving platform to change the motion types. By adding a rTPS limb which has two phases, a new metamorphic parallel mechanism is introduced in [21]. The link-coincidence-based geometric-constraint method is proposed in [22] to obtain reconfigurable mechanisms originated from carton folds and packaging dated back to 1996. At the same year, Wohlhart in [23] showed mechanisms that changed mobility through singularities.

In [24], Li and Hervé investigated several PM with two distinct Schönflies modes. The

\footnotetext{
${ }^{1} \mathrm{R}, \mathrm{P}, \mathrm{E}, \mathrm{U}, \mathrm{S}$ denote revolute, prismatic, planar, universal and spherical joints, respectively.
} 
Schönflies motion contains three independent translations and one pure rotation about an axis of fixed direction, namely 3T1R. The authors continued in [25] to present the systematic approach to synthesize the iso-constrained parallel Schönflies motion generators with two identical 5-dof limbs.

The type synthesis of the 3T1R PM with four identical limb structures was performed in [26], which leads to a kinematic architecture with four revolute actuators, namely the 4RUU PM. In [27], eight solutions of the direct kinematics were enumerated by using the linear implicitization algorithm. Amine et al. in [28, 29] investigated the singularity conditions of the 4-RUU PM by using the Grassmann-Cayley Algebra and the Grassmann Geometry. It is shown that the 4-RUU PM is an over-constrained manipulator and it shares some common properties among the constraint wrenches.

By using an algebraic description of the manipulator and the Study's kinematic mapping based upon [30], a characterization of the operation modes of the 4-RUU PM are discussed in more details in this paper. Due to the unique topology of the RUU limb that comprises two links with one revolute actuator attached to the base, the actuated joint angle always appears in every constraint equation. This kinematic issue does not allow to compute a primary decomposition because the constraint equation changes for every joint inputs. As a consequence, the 4-RUU PM is decomposed into two iso-constrained 2-RUU PM. The constraint equations of each 2-RUU PM are initially derived and the primary decomposition is computed. It turns out that the 2-RUU PM has three 4-dof operation modes. By combining the results of primary decomposition from both 2-RUU PM, the operation modes of the 4-RUU PM can be characterized. It reveals that the 4-RUU PM has two 4-dof operation modes and one 2-dof operation mode. The singularities are examined by deriving the determinant of the Jacobian matrix of the constraint equations with respect to the Study parameters. It is shown that the manipulators are able to change from one operation mode to another operation mode by passing through the configurations that belong to both modes. The singularity conditions are mapped onto the joint space. Eventually, the changes of operation modes are illustrated.

This paper is organized as follows: A detailed description of the manipulator architecture is given in Section 2. The constraint equations of the manipulators are expressed in Section 3. These constraint equations are used to identify the operation modes in Section 4. In Section 5 , the singularity conditions and self-motions are presented. Eventually, the operation modes changing of the 4-RUU PM is discussed in Section 6. 


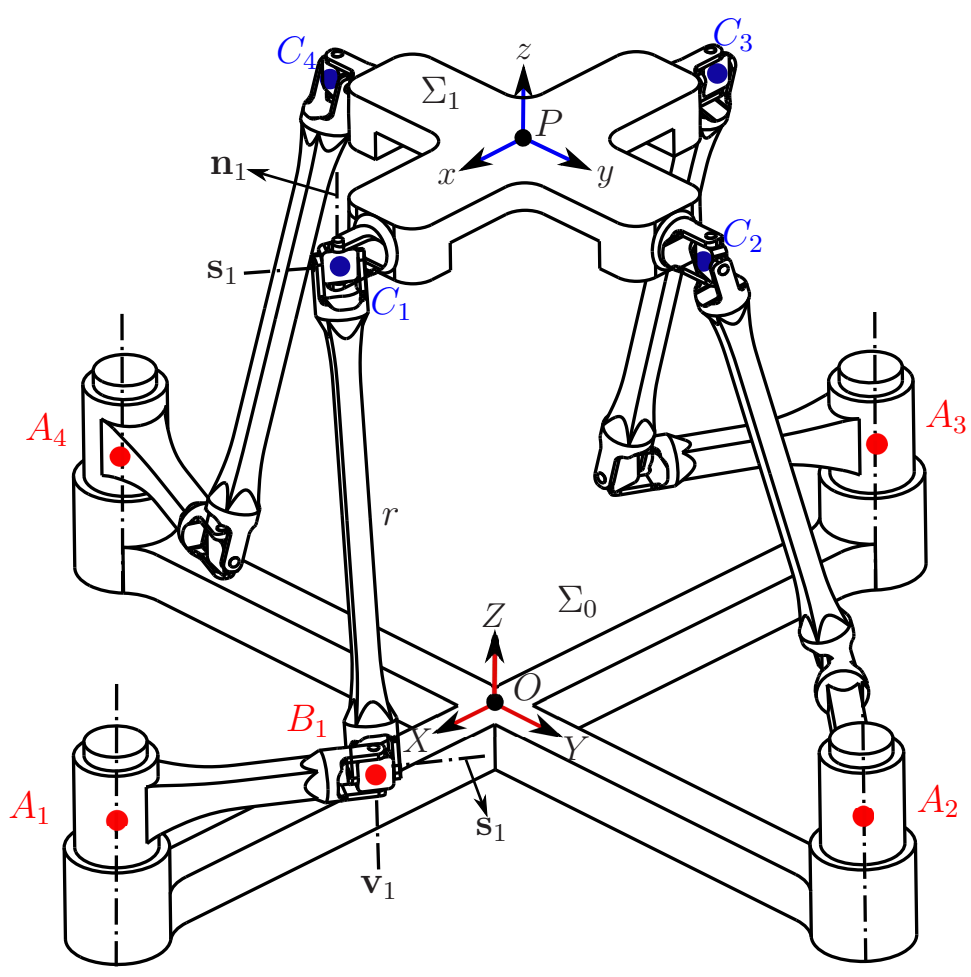

Figure 1: The 4-RUU PM.

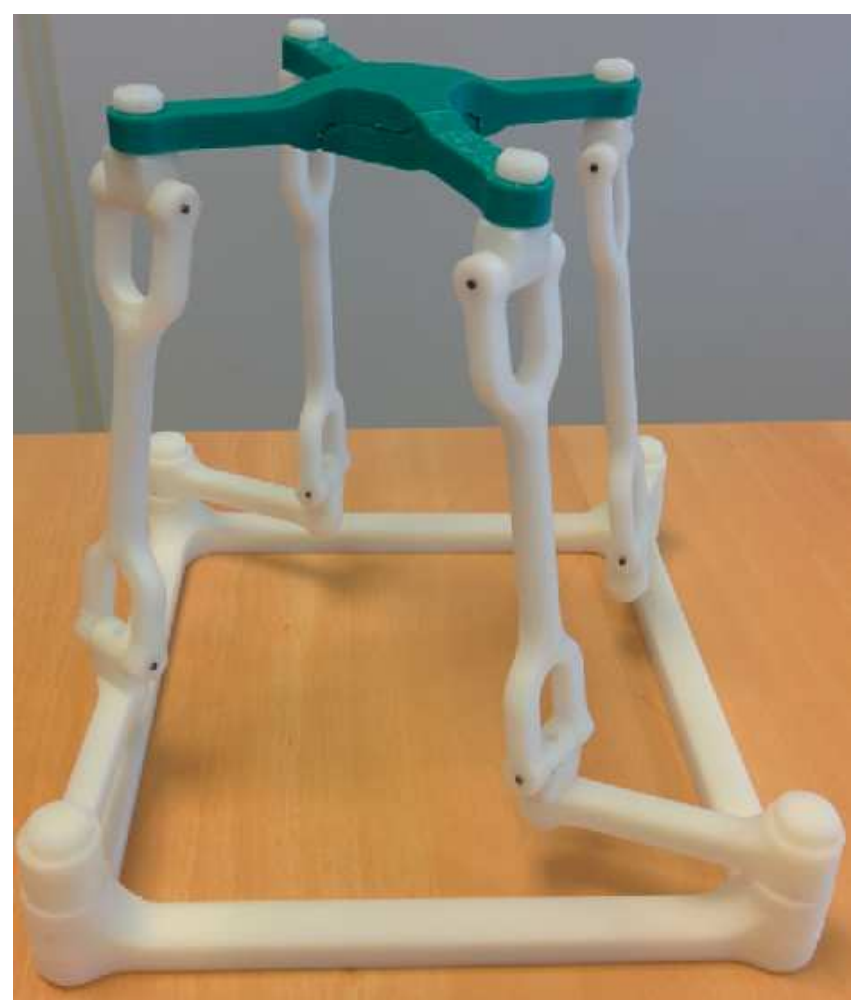

Figure 2: Printed model of 4-RUU PM. 


\section{Manipulator architecture}

The 4-RUU PM shown in Fig. 1, is composed of a square base, a square moving platform, and four identical limbs. The origin $O$ of the fixed frame $\Sigma_{0}$ and the origin $P$ of the moving frame $\Sigma_{1}$ are located at the center of the square base and the square moving platform, respectively. Each limb is composed of five R-joints such that the second and the third ones, as well as the forth and the fifth ones, are built with intersecting and perpendicular axes. Thus they are assimilated to $\mathrm{U}$-joint. The printed model of this manipulator is shown in Fig. 2.

The first R-joint is attached to the base and is actuated. Its rotation angle is defined by $\theta_{1 i}$ $(i=1, \ldots, 4)$. The axes of the first and the second joints are directed along $Z$-axis. The axis of the fifth joint is directed along $z$-axis. The second axis and the fifth axis are denoted by $\mathbf{v}_{i}$ and $\mathbf{n}_{i}(i=1, \ldots, 4)$, respectively. The axes of the third and the forth joints are parallel. The axis of the third joint is denoted by $\mathbf{s}_{i}(i=1, \ldots, 4)$ and it changes instantaneously as a function of $\theta_{2 i}(i=1 . .4)$ as shown in Fig. 3, hence:

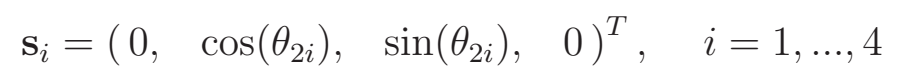

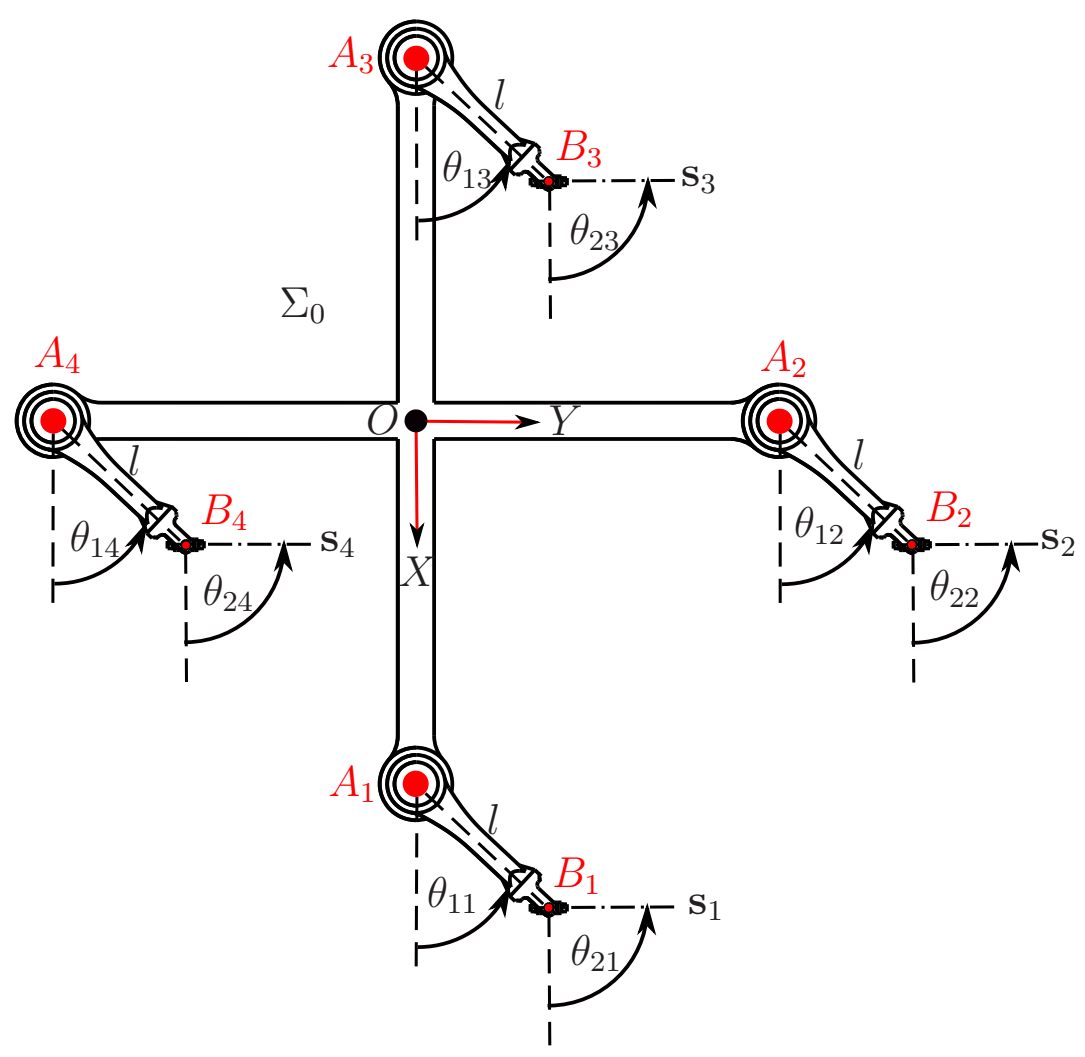

Figure 3: Parametrization of the first two joint angles in each leg from top view. 
The first R-joint of the $i$-th limb is located at point $A_{i}$ with distance $a$ from the origin $O$ of the fixed frame $\Sigma_{0}$. The first $\mathrm{U}$-joint is denoted by point $B_{i}$ with distance $l$ from point $A_{i}$. Link $A_{i} B_{i}$ always moves in a plane normal to $\mathbf{v}_{i}$. Hence the coordinates of points $A_{i}$ and $B_{i}$ expressed in the fixed frame $\Sigma_{0}$ are:

$$
\begin{aligned}
& \mathbf{r}_{A_{1}}^{0}=\left(\begin{array}{llll}
1, & a, & 0, & 0
\end{array}\right)^{T} \quad \mathbf{r}_{B_{1}}^{0}=\left(\begin{array}{lll}
1, & l \cos \left(\theta_{11}\right)+a, \quad l \sin \left(\theta_{11}\right), \quad 0
\end{array}\right)^{T} \\
& \mathbf{r}_{A_{2}}^{0}=\left(\begin{array}{llll}
1, & 0, & a, & 0
\end{array}\right)^{T} \quad \mathbf{r}_{B_{2}}^{0}=\left(\begin{array}{lll}
1, & l \cos \left(\theta_{12}\right), \quad l \sin \left(\theta_{12}\right)+a, \quad 0
\end{array}\right)^{T} \\
& \mathbf{r}_{A_{3}}^{0}=\left(\begin{array}{llll}
1, & -a, & 0, & 0
\end{array}\right)^{T} \quad \mathbf{r}_{B_{3}}^{0}=\left(\begin{array}{ll}
1, & l \cos \left(\theta_{13}\right)-a, \quad l \sin \left(\theta_{13}\right), \quad 0
\end{array}\right)^{T} \\
& \mathbf{r}_{A_{4}}^{0}=\left(\begin{array}{llll}
1, & 0, & -a, & 0
\end{array}\right)^{T} \quad \mathbf{r}_{B_{4}}^{0}=\left(\begin{array}{lll}
1, & l \cos \left(\theta_{14}\right), \quad l \sin \left(\theta_{14}\right)-a, \quad 0
\end{array}\right)^{T}
\end{aligned}
$$

The moving platform is connected to the limbs by four U-joints, of which the intersection point of the R-joint axes is denoted by $C_{i}$. The length of the moving platform from the origin $P$ of the moving frame $\Sigma_{1}$ to point $C_{i}$ is defined by $b$. The length of link $B_{i} C_{i}$ is defined by $r$. The coordinates of point $C_{i}$ expressed in the moving frame $\Sigma_{1}$ are:

$$
\begin{array}{lll}
\mathbf{r}_{C_{1}}^{1}=\left(\begin{array}{llll}
1, & b, & 0, & 0
\end{array}\right)^{T} & \mathbf{r}_{C_{3}}^{1}=\left(\begin{array}{lllll}
1, & -b, & 0, & 0
\end{array}\right)^{T} \\
\mathbf{r}_{C_{2}}^{1}=\left(\begin{array}{llllll}
1, & 0, & b, & 0
\end{array}\right)^{T} & \mathbf{r}_{C_{4}}^{1}=\left(\begin{array}{llll}
1, & 0, & -b, & 0
\end{array}\right)^{T}
\end{array}
$$

As a consequence, there are four design parameters $a, b, l$, and $r$; and four joint variables $\theta_{11}, \theta_{12}, \theta_{13}$, and $\theta_{14}$ that determine the motions of the 4-RUU PM.

\section{Constraint equations}

In this section, the constraint equations are derived whose solutions illustrate the possible poses of the moving platform (coordinate frame $\Sigma_{1}$ ) with respect to $\Sigma_{0}$. To obtain the coordinates of points $C_{i}$ and vectors $\mathbf{n}_{i}$ expressed in $\Sigma_{0}$, the Study parametrization of a spatial Euclidean transformation matrix $\mathbf{M} \in S E(3)$ based on [30] is used.

$$
\mathbf{M}=\left(\begin{array}{cc}
x_{0}^{2}+x_{1}^{2}+x_{2}^{2}+x_{3}^{2} & \mathbf{0}_{3 \times 1}^{T} \\
\mathbf{M}_{T} & \mathbf{M}_{R}
\end{array}\right)
$$

where $\mathbf{M}_{T}$ and $\mathbf{M}_{R}$ represent the translational and rotational parts of the transformation matrix $\mathbf{M}$, respectively, and are expressed as follows:

$$
\begin{gathered}
\mathbf{M}_{T}=\left(\begin{array}{c}
2\left(-x_{0} y_{1}+x_{1} y_{0}-x_{2} y_{3}+x_{3} y_{2}\right) \\
2\left(-x_{0} y_{2}+x_{1} y_{3}+x_{2} y_{0}-x_{3} y_{1}\right) \\
2\left(-x_{0} y_{3}-x_{1} y_{2}+x_{2} y_{1}+x_{3} y_{0}\right)
\end{array}\right) \\
\mathbf{M}_{R}=\left(\begin{array}{ccc}
x_{0}^{2}+x_{1}^{2}-x_{2}^{2}-x_{3}^{2} & 2\left(x_{1} x_{2}-x_{0} x_{3}\right) & 2\left(x_{1} x_{3}+x_{0} x_{2}\right) \\
2\left(x_{1} x_{2}+x_{0} x_{3}\right) & x_{0}^{2}-x_{1}^{2}+x_{2}^{2}-x_{3}^{2} & 2\left(x_{2} x_{3}-x_{0} x_{1}\right) \\
2\left(x_{1} x_{3}-x_{0} x_{2}\right) & 2\left(x_{2} x_{3}+x_{0} x_{1}\right) & x_{0}^{2}-x_{1}^{2}-x_{2}^{2}+x_{3}^{2}
\end{array}\right)
\end{gathered}
$$


The parameters $x_{0}, x_{1}, x_{2}, x_{3}, y_{0}, y_{1}, y_{2}, y_{3}$, which appear in matrix $\mathbf{M}$, are called Study parameters. These parameters make it possible to parametrize $S E(3)$ with dual quaternions. The Study's kinematic mapping maps each spatial Euclidean displacement of $S E(3)$ via transformation matrix $\mathbf{M}$ onto a projective point $X\left[x_{0}: x_{1}: x_{2}: x_{3}: y_{0}: y_{1}: y_{2}: y_{3}\right]$ in the 6-dimensional Study quadric $S \in \mathbb{P}^{7}[6]$, such that:

$$
\begin{gathered}
S E(3) \rightarrow X \in \mathbb{P}^{7} \\
\left(x_{0}: x_{1}: x_{2}: x_{3}: y_{0}: y_{1}: y_{2}: y_{3}\right)^{T} \neq(0: 0: 0: 0: 0: 0: 0: 0)^{T}
\end{gathered}
$$

Every projective point $X$ will represent a spatial Euclidean displacement, if it fulfils the following equation and inequality:

$$
\begin{gathered}
x_{0} y_{0}+x_{1} y_{1}+x_{2} y_{2}+x_{3} y_{3}=0, \\
x_{0}^{2}+x_{1}^{2}+x_{2}^{2}+x_{3}^{2} \neq 0
\end{gathered}
$$

Those two conditions will be used in the following computations to simplify the algebraic expressions. First of all, the tangent half-angle substitutions are performed to rewrite the trigonometric functions of $\theta_{1 i}$ and $\theta_{2 i}(i=1, \ldots, 4)$ in terms of rational functions of a new variable $t_{i j}$. However, the tangent half-angle substitutions will increase the degree of variable and make the computation quite heavy.

$$
\cos \left(\theta_{i j}\right)=\frac{1-t_{i j}^{2}}{1+t_{i j}^{2}}, \quad \sin \left(\theta_{i j}\right)=\frac{2 t_{i j}^{2}}{1+t_{i j}^{2}}, \quad i=1,2, \quad j=1, \ldots, 4
$$

where $t_{i j}=\tan \left(\frac{\theta_{i j}}{2}\right)$. The coordinates of points $C_{i}$ and vectors $\mathbf{n}_{i}$ expressed in the fixed frame $\Sigma_{0}$ are obtained by:

$$
\mathbf{r}_{C_{i}}^{0}=\mathbf{M} \mathbf{r}_{C_{i}}^{1}, \quad \mathbf{n}_{i}^{0}=\mathbf{M} \mathbf{n}_{i}^{1}, \quad i=1, \ldots, 4
$$

The coordinates of all points are given in terms of the Study parameters and the design parameters. The constraint equations can be obtained by examining the design of RUU limb. The link connecting points $B_{i}$ and $C_{i}$ is coplanar to the vectors $\mathbf{v}_{i}$ and $\mathbf{n}_{i}^{0}$. Accordingly, the scalar triple product of vectors $\left(\mathbf{r}_{C_{i}}^{0}-\mathbf{r}_{B_{i}}^{0}\right), \mathbf{v}_{i}$ and $\mathbf{n}_{i}^{0}$ vanishes, namely:

$$
\left(\mathbf{r}_{C_{i}}^{0}-\mathbf{r}_{B_{i}}^{0}\right)^{T} .\left(\mathbf{v}_{i} \times \mathbf{n}_{i}^{0}\right)=0 \quad, \quad i=1, \ldots, 4
$$

After computing the corresponding scalar triple product and removing the common denominators, the following constraint equations come out: 
$g_{1}:\left(a t_{11}^{2}-b t_{11}^{2}-l t_{11}^{2}+a-b+l\right) x_{0} x_{1}+2 l t_{11} x_{0} x_{2}-\left(2 t_{11}^{2}+2\right) x_{0} y_{0}+2 l t_{11} x_{3} x_{1}+$ $\left(-a t_{11}^{2}-b t_{11}^{2}+l t_{11}^{2}-a-b-l\right) x_{3} x_{2}+\left(-2 t_{11}^{2}-2\right) y_{3} x_{3}=0$

$g_{2}:\left(l-l t_{12}^{2}\right) x_{0} x_{1}+\left(a t_{12}^{2}-b t_{12}^{2}+2 l t_{12}+a-b\right) x_{0} x_{2}-\left(2 t_{12}^{2}+2\right) x_{0} y_{0}+\left(a t_{12}^{2}+b t_{12}^{2}\right.$ $\left.+2 l t_{12}+a+b\right) x_{3} x_{1}+\left(l t_{12}^{2}-l\right) x_{3} x_{2}-\left(2 t_{12}^{2}+2\right) y_{3} x_{3}=0$

$g_{3}:\left(a t_{13}^{2}-b t_{13}^{2}+l t_{13}^{2}+a-b-l\right) x_{0} x_{1}-2 l t_{13} x_{0} x_{2}+\left(2 t_{13}^{2}+2\right) x_{0} y_{0}-2 l t_{13} x_{1} x_{3}+$ $\left(-a t_{13}^{2}-b t_{13}^{2}-l t_{13}^{2}-a-b+l\right) x_{2} x_{3}+\left(2 t_{13}^{2}+2\right) x_{3} y_{3}=0$

$g_{4}: \quad\left(l t_{14}^{2}-l\right) x_{0} x_{1}+\left(a t_{14}^{2}-b t_{14}^{2}-2 l t_{14}+a-b\right) x_{0} x_{2}+\left(2 t_{14}^{2}+2\right) x_{0} y_{0}+\left(a t_{14}^{2}+b t_{14}^{2}\right.$ $\left.-2 l t_{14}+a+b\right) x_{1} x_{3}+\left(-l t_{14}^{2}+l\right) x_{2} x_{3}+\left(2 t_{14}^{2}+2\right) x_{3} y_{3}=0$

To derive the constraint equations corresponding to the link length $r$ of link $B_{i} C_{i}$, the distance equation can be formulated as: $\left\|\left(\mathbf{r}_{C_{i}}^{0}-\mathbf{r}_{B_{i}}^{0}\right)\right\|^{2}=r^{2}$. As a consequence, the following four equations are obtained:

$g_{5}: \quad\left(a^{2} t_{11}^{2}-2 a b t_{11}^{2}-2 a l t_{11}^{2}+b^{2} t_{11}^{2}+2 b l t_{11}^{2}+l^{2} t_{11}^{2}-r^{2} t_{11}^{2}+a^{2}-2 a b+2 a l+b^{2}-2 b l+l^{2}-\right.$ $\left.r^{2}\right) x_{0}^{2}-8 b l t_{11} x_{0} x_{3}+\left(4 a t_{11}^{2}-4 b t_{11}^{2}-4 l t_{11}^{2}+4 a-4 b+4 l\right) x_{0} y_{1}+8 l t_{11} x_{0} y_{2}+\left(a^{2} t_{11}^{2}-2 a\right.$ $\left.b t_{11}^{2}-2 a l t_{11}^{2}+b^{2} t_{11}^{2}+2 b l t_{11}^{2}+l^{2} t_{11}^{2}-r^{2} t_{11}^{2}+a^{2}-2 a b+2 a l+b^{2}-2 b l+l^{2}-r^{2}\right) x_{1}^{2}-8$ $b l t_{11} x_{1} x_{2}+\left(-4 a t_{11}^{2}+4 b t_{11}^{2}+4 l t_{11}^{2}-4 a+4 b-4 l\right) x_{1} y_{0}-8 l t_{11} x_{1} y_{3}+\left(a^{2} t_{11}^{2}+2 a b t_{11}^{2}-2\right.$ $\left.a l t_{11}^{2}+b^{2} t_{11}^{2}-2 b l t_{11}^{2}+l^{2} t_{11}^{2}-r^{2} t_{11}^{2}+a^{2}+2 a b+2 a l+b^{2}+2 b l+l^{2}-r^{2}\right) x_{2}^{2}-8 l t_{11} x_{2} y_{0}$ $+\left(4 a t_{11}^{2}+4 b t_{11}^{2}-4 l t_{11}^{2}+4 a+4 b+4 l\right) x_{2} y_{3}+\left(a^{2} t_{11}^{2}+2 a b t_{11}^{2}-2 a l t_{11}^{2}+b^{2} t_{11}^{2}-2 b l t_{11}^{2}+\right.$ $\left.l^{2} t_{11}^{2}-r^{2} t_{11}^{2}+a^{2}+2 a b+2 a l+b^{2}+2 b l+l^{2}-r^{2}\right) x_{3}^{2}+8 l t_{11} x_{3} y_{1}+\left(-4 a t_{11}^{2}-4 b t_{11}^{2}+4 l\right.$ $\left.t_{11}^{2}-4 a-4 b-4 l\right) x_{3} y_{2}+\left(4 t_{11}^{2}+4\right) y_{0}^{2}+\left(4 t_{11}^{2}+4\right) y_{1}^{2}+\left(4 t_{11}^{2}+4\right) y_{2}^{2}+\left(4 t_{11}^{2}+4\right) y_{3}^{2}=0$

$g_{6}: \quad\left(a^{2} t_{12}^{2}-2 a b t_{12}^{2}+b^{2} t_{12}^{2}+l^{2} t_{12}^{2}-r^{2} t_{12}^{2}+4 a l t_{12}-4 b l t_{12}+a^{2}-2 a b+b^{2}+l^{2}-r^{2}\right) x_{0}^{2}+(-$ $\left.4 b l t_{12}^{2}+4 b l\right) x_{0} x_{3}+\left(-4 l t_{12}^{2}+4 l\right) x_{0} y_{1}+\left(4 a t_{12}^{2}-4 b t_{12}^{2}+8 l t_{12}+4 a-4 b\right) x_{0} y_{2}+\left(a^{2} t_{12}^{2}+2\right.$ $\left.a b t_{12}^{2}+b^{2} t_{12}^{2}+l^{2} t_{12}^{2}-r^{2} t_{12}^{2}+4 a l t_{12}+4 b l t_{12}+a^{2}+2 a b+b^{2}+l^{2}-r^{2}\right) x_{1}^{2}+\left(4 b l t_{12}^{2}-4 b l\right)$ $x_{1} x_{2}+\left(4 l t_{12}^{2}-4 l\right) x_{1} y_{0}+\left(-4 a t_{12}^{2}-4 b t_{12}^{2}-8 l t_{12}-4 a-4 b\right) x_{1} y_{3}+\left(a^{2} t_{12}^{2}-2 a b t_{12}^{2}+b^{2} t_{12}^{2}\right.$ $\left.+l^{2} t_{12}^{2}-r^{2} t_{12}^{2}+4 a l t_{12}-4 b l t_{12}+a^{2}-2 a b+b^{2}+l^{2}-r^{2}\right) x_{2}^{2}+\left(-4 a t_{12}^{2}+4 b t_{12}^{2}-8 l t_{12}-4\right.$ $a+4 b) x_{2} y_{0}+\left(-4 l t_{12}^{2}+4 l\right) x_{2} y_{3}+\left(a^{2} t_{12}^{2}+2 a b t_{12}^{2}+b^{2} t_{12}^{2}+l^{2} t_{12}^{2}-r^{2} t_{12}^{2}+4 a l t_{12}+4 b l t_{12}\right.$ $\left.+a^{2}+2 a b+b^{2}+l^{2}-r^{2}\right) x_{3}^{2}+\left(4 a t_{12}^{2}+4 b t_{12}^{2}+8 l t_{12}+4 a+4 b\right) x_{3} y_{1}+\left(4 l t_{12}^{2}-4 l\right) x_{3} y_{2}+$ $\left(4 t_{12}^{2}+4\right) y_{0}^{2}+\left(4 t_{12}^{2}+4\right) y_{1}^{2}+\left(4 t_{12}^{2}+4\right) y_{2}^{2}+\left(4 t_{12}^{2}+4\right) y_{3}^{2}=0$ 
$g_{7}:\left(a^{2} t_{13}^{2}-2 a b t_{13}^{2}+2 a l t_{13}^{2}+b^{2} t_{13}^{2}-2 b l t_{13}^{2}+l^{2} t_{13}^{2}-r^{2} t_{13}^{2}+a^{2}-2 a b-2 a l+b^{2}+2 b l+l^{2}-\right.$ $\left.r^{2}\right) x_{0}^{2}+8 b l t_{13} x_{0} x_{3}+\left(-4 a t_{13}^{2}+4 b t_{13}^{2}-4 l t_{13}^{2}-4 a+4 b+4 l\right) x_{0} y_{1}+8 l t_{13} x_{0} y_{2}+\left(a^{2} t_{13}^{2}-2\right.$ $\left.a b t_{13}^{2}+2 a l t_{13}^{2}+b^{2} t_{13}^{2}-2 b l t_{13}^{2}+l^{2} t_{13}^{2}-r^{2} t_{13}^{2}+a^{2}-2 a b-2 a l+b^{2}+2 b l+l^{2}-r^{2}\right) x_{1}^{2}+8$ $b l t_{13} x_{1} x_{2}+\left(4 a t_{13}^{2}-4 b t_{13}^{2}+4 l t_{13}^{2}+4 a-4 b-4 l\right) x_{1} y_{0}-8 l t_{13} x_{1} y_{3}+\left(a^{2} t_{13}^{2}+2 a b t_{13}^{2}+2 a l\right.$ $\left.t_{13}^{2}+b^{2} t_{13}^{2}+2 b l t_{13}^{2}+l^{2} t_{13}^{2}-r^{2} t_{13}^{2}+a^{2}+2 a b-2 a l+b^{2}-2 b l+l^{2}-r^{2}\right) x_{2}^{2}-8 l t_{13} x_{2} y_{0}+($ $\left.-4 a t_{13}^{2}-4 b t_{13}^{2}-4 l t_{13}^{2}-4 a-4 b+4 l\right) x_{2} y_{3}+\left(a^{2} t_{13}^{2}+2 a b t_{13}^{2}+2 a l t_{13}^{2}+b^{2} t_{13}^{2}+2 b l t_{13}^{2}+l^{2}\right.$ $\left.t_{13}^{2}-r^{2} t_{13}^{2}+a^{2}+2 a b-2 a l+b^{2}-2 b l+l^{2}-r^{2}\right) x_{3}^{2}+8 l t_{13} x_{3} y_{1}+\left(4 a t_{13}^{2}+4 b t_{13}^{2}+4 l t_{13}^{2}+\right.$ $4 a+4 b-4 l) x_{3} y_{2}+\left(4 t_{13}^{2}+4\right) y_{0}^{2}+\left(4 t_{13}^{2}+4\right) y_{1}^{2}+\left(4 t_{13}^{2}+4\right) y_{2}^{2}+\left(4 t_{13}^{2}+4\right) y_{3}^{2}=0$

$g_{8}: \quad\left(a^{2} t_{14}^{2}-2 a b t_{14}^{2}+b^{2} t_{14}^{2}+l^{2} t_{14}^{2}-r^{2} t_{14}^{2}-4 a l t_{14}+4 b l t_{14}+a^{2}-2 a b+b^{2}+l^{2}-r^{2}\right) x_{0}^{2}+(-$ $\left.4 b l+4 b l t_{14}^{2}\right) x_{0} x_{3}+\left(-4 l t_{14}^{2}+4 l\right) x_{0} y_{1}+\left(-4 a t_{14}^{2}+4 b t_{14}^{2}+8 l t_{14}-4 a+4 b\right) x_{0} y_{2}+\left(a^{2} t_{14}^{2}+\right.$ $\left.2 a b t_{14}^{2}+b^{2} t_{14}^{2}+l^{2} t_{14}^{2}-r^{2} t_{14}^{2}-4 a l t_{14}-4 b l t_{14}+a^{2}+2 a b+b^{2}+l^{2}-r^{2}\right) x_{1}^{2}+\left(-4 b l t_{14}^{2}+4\right.$ bl) $x_{1} x_{2}+\left(4 l t_{14}^{2}-4 l\right) x_{1} y_{0}+\left(4 a t_{14}^{2}+4 b t_{14}^{2}-8 l t_{14}+4 a+4 b\right) x_{1} y_{3}+\left(a^{2} t_{14}^{2}-2 a b t_{14}^{2}+b^{2} t_{14}^{2}\right.$ $\left.+l^{2} t_{14}^{2}-r^{2} t_{14}^{2}-4 a l t_{14}+4 b l t_{14}+a^{2}-2 a b+b^{2}+l^{2}-r^{2}\right) x_{2}^{2}+\left(4 a t_{14}^{2}-4 b t_{14}^{2}-8 l t_{14}+4 a\right.$ $-4 b) x_{2} y_{0}+\left(-4 l t_{14}^{2}+4 l\right) x_{2} y_{3}+\left(a^{2} t_{14}^{2}+2 a b t_{14}^{2}+b^{2} t_{14}^{2}+l^{2} t_{14}^{2}-r^{2} t_{14}^{2}-4 a l t_{14}-4 b l t_{14}+\right.$ $\left.a^{2}+2 a b+b^{2}+l^{2}-r^{2}\right) x_{3}^{2}+\left(-4 a t_{14}^{2}-4 b t_{14}^{2}+8 l t_{14}-4 a-4 b\right) x_{3} y_{1}+\left(4 l t_{14}^{2}-4 l\right) x_{3} y_{2}+$ $\left(4 t_{14}^{2}+4\right) y_{0}^{2}+\left(4 t_{14}^{2}+4\right) y_{1}^{2}+\left(4 t_{14}^{2}+4\right) y_{2}^{2}+\left(4 t_{14}^{2}+4\right) y_{3}^{2}=0$

To derive the constraint equations corresponding to the axes $\mathbf{s}_{i}$ of each limb, the scalar product of vector $\overrightarrow{B_{i} C_{i}}$ and vector $\mathbf{s}_{i}$ should vanish, as : $\left(\mathbf{r}_{C_{i}}^{0}-\mathbf{r}_{B_{i}}^{0}\right)^{T} \mathbf{s}_{i}=0$. Hence, the following constraint equations are obtained:

$g_{9}:\left(-a t_{11}^{2} t_{21}^{2}+b t_{11}^{2} t_{21}^{2}+l t_{11}^{2} t_{21}^{2}+a t_{11}^{2}-a t_{21}^{2}-b t_{11}^{2}+b t_{21}^{2}-l t_{11}^{2}+4 l t_{11} t_{21}-l t_{21}^{2}+a-b+l\right)$ $x_{0}^{2}+\left(-4 b t_{11}^{2} t_{21}-4 b t_{21}\right) x_{3} x_{0}+\left(-2 t_{11}^{2} t_{21}^{2}+2 t_{11}^{2}-2 t_{21}^{2}+2\right) x_{0} y_{1}+\left(4 t_{11}^{2} t_{21}+4 t_{21}\right) x_{0} y_{2}+$ $\left(-a t_{11}^{2} t_{21}^{2}+b t_{11}^{2} t_{21}^{2}+l t_{11}^{2} t_{21}^{2}+a t_{11}^{2}-a t_{21}^{2}-b t_{11}^{2}+b t_{21}^{2}-l t_{11}^{2}+4 l t_{11} t_{21}-l t_{21}^{2}+a-b+l\right)$ $x_{1}^{2}+\left(-4 b t_{11}^{2} t_{21}-4 b t_{21}\right) x_{2} x_{1}+\left(2 t_{11}^{2} t_{21}^{2}-2 t_{11}^{2}+2 t_{21}^{2}-2\right) x_{1} y_{0}+\left(-4 t_{11}^{2} t_{21}-4 t_{21}\right) x_{1} y_{3}+$ $\left(-a t_{11}^{2} t_{21}^{2}-b t_{11}^{2} t_{21}^{2}+l t_{11}^{2} t_{21}^{2}+a t_{11}^{2}-a t_{21}^{2}+b t_{11}^{2}-b t_{21}^{2}-l t_{11}^{2}+4 l t_{11} t_{21}-l t_{21}^{2}+a+b+l\right)$ $x_{2}^{2}+\left(-4 t_{11}^{2} t_{21}-4 t_{21}\right) x_{2} y_{0}+\left(-2 t_{11}^{2} t_{21}^{2}+2 t_{11}^{2}-2 t_{21}^{2}+2\right) x_{2} y_{3}+\left(-a t_{11}^{2} t_{21}^{2}-b t_{11}^{2} t_{21}^{2}+l t_{11}^{2}\right.$ $\left.t_{21}^{2}+a t_{11}^{2}-a t_{21}^{2}+b t_{11}^{2}-b t_{21}^{2}-l t_{11}^{2}+4 l t_{11} t_{21}-l t_{21}^{2}+a+b+l\right) x_{3}^{2}+\left(4 t_{11}^{2} t_{21}+4 t_{21}\right) x_{3} y_{1}$ $+\left(2 t_{11}^{2} t_{21}^{2}-2 t_{11}^{2}+2 t_{21}^{2}-2\right) x_{3} y_{2}=0$ 


$$
\begin{aligned}
g_{10}: & \left(l t_{12}^{2} t_{22}^{2}+2 a t_{12}^{2} t_{22}-2 b t_{12}^{2} t_{22}-l t_{12}^{2}+4 l t_{12} t_{22}-l t_{22}^{2}+2 a t_{22}-2 b t_{22}+l\right) x_{0}^{2}+\left(-2 b t_{12}^{2} t_{22}^{2}+2\right. \\
& \left.b t_{12}^{2}-2 b t_{22}^{2}+2 b\right) x_{0} x_{3}+\left(-2 t_{12}^{2} t_{22}^{2}+2 t_{12}^{2}-2 t_{22}^{2}+2\right) x_{0} y_{1}+\left(4 t_{12}^{2} t_{22}+4 t_{22}\right) x_{0} y_{2}+\left(l t_{12}^{2} t_{22}^{2}+\right. \\
& \left.2 a t_{12}^{2} t_{22}+2 b t_{12}^{2} t_{22}-l t_{12}^{2}+4 l t_{12} t_{22}-l t_{22}^{2}+2 a t_{22}+2 b t_{22}+l\right) x_{1}^{2}+\left(2 b t_{12}^{2} t_{22}^{2}-2 b t_{12}^{2}+2 b t_{22}^{2}\right. \\
& -2 b) x_{1} x_{2}+\left(2 t_{12}^{2} t_{22}^{2}-2 t_{12}^{2}+2 t_{22}^{2}-2\right) x_{1} y_{0}+\left(-4 t_{12}^{2} t_{22}-4 t_{22}\right) x_{1} y_{3}+\left(l t_{12}^{2} t_{22}^{2}+2 a t_{12}^{2} t_{22}-\right. \\
& \left.2 b t_{12}^{2} t_{22}-l t_{12}^{2}+4 l t_{12} t_{22}-l t_{22}^{2}+2 a t_{22}-2 b t_{22}+l\right) x_{2}^{2}+\left(-4 t_{12}^{2} t_{22}-4 t_{22}\right) x_{2} y_{0}+\left(-2 t_{12}^{2} t_{22}^{2}\right. \\
& \left.+2 t_{12}^{2}-2 t_{22}^{2}+2\right) x_{2} y_{3}+\left(l t_{12}^{2} t_{22}^{2}+2 a t_{12}^{2} t_{22}+2 b t_{12}^{2} t_{22}-l t_{12}^{2}+4 l t_{12} t_{22}-l t_{22}^{2}+2 a t_{22}+2 b\right. \\
& \left.t_{22}+l\right) x_{3}^{2}+\left(4 t_{12}^{2} t_{22}+4 t_{22}\right) x_{3} y_{1}+\left(2 t_{12}^{2} t_{22}^{2}-2 t_{12}^{2}+2 t_{22}^{2}-2\right) x_{3} y_{2}=0
\end{aligned}
$$

$g_{11}:\left(a t_{13}^{2} t_{23}^{2}-b t_{13}^{2} t_{23}^{2}+l t_{13}^{2} t_{23}^{2}-a t_{13}^{2}+a t_{23}^{2}+b t_{13}^{2}-b t_{23}^{2}-l t_{13}^{2}+4 l t_{13} t_{23}-l t_{23}^{2}-a+b+l\right) x_{0}^{2}$ $+\left(4 b t_{13}^{2} t_{23}+4 b t_{23}\right) x_{0} x_{3}+\left(-2 t_{13}^{2} t_{23}^{2}+2 t_{13}^{2}-2 t_{23}^{2}+2\right) x_{0} y_{1}+\left(4 t_{13}^{2} t_{23}+4 t_{23}\right) x_{0} y_{2}+\left(a t_{13}^{2} t_{23}^{2}\right.$ $\left.-b t_{13}^{2} t_{23}^{2}+l t_{13}^{2} t_{23}^{2}-a t_{13}^{2}+a t_{23}^{2}+b t_{13}^{2}-b t_{23}^{2}-l t_{13}^{2}+4 l t_{13} t_{23}-l t_{23}^{2}-a+b+l\right) x_{1}^{2}+\left(4 b t_{13}^{2}\right.$ $\left.t_{23}+4 b t_{23}\right) x_{1} x_{2}+\left(2 t_{13}^{2} t_{23}^{2}-2 t_{13}^{2}+2 t_{23}^{2}-2\right) x_{1} y_{0}+\left(-4 t_{13}^{2} t_{23}-4 t_{23}\right) x_{1} y_{3}+\left(a t_{13}^{2} t_{23}^{2}+b t_{13}^{2}\right.$ $\left.t_{23}^{2}+l t_{13}^{2} t_{23}^{2}-a t_{13}^{2}+a t_{23}^{2}-b t_{13}^{2}+b t_{23}^{2}-l t_{13}^{2}+4 l t_{13} t_{23}-l t_{23}^{2}-a-b+l\right) x_{2}^{2}-\left(4 t_{13}^{2} t_{23}+4\right.$ $\left.t_{23}\right) x_{2} y_{0}+\left(-2 t_{13}^{2} t_{23}^{2}+2 t_{13}^{2}-2 t_{23}^{2}+2\right) x_{2} y_{3}+\left(a t_{13}^{2} t_{23}^{2}+b t_{13}^{2} t_{23}^{2}+l t_{13}^{2} t_{23}^{2}-a t_{13}^{2}+a t_{23}^{2}-b t_{13}^{2}\right.$ $\left.+b t_{23}^{2}-l t_{13}^{2}+4 l t_{13} t_{23}-l t_{23}^{2}-a-b+l\right) x_{3}^{2}+\left(4 t_{13}^{2} t_{23}+4 t_{23}\right) x_{3} y_{1}+\left(2 t_{13}^{2} t_{23}^{2}-2 t_{13}^{2}+2 t_{23}^{2}\right.$ $-2) x_{3} y_{2}=0$

$g_{12}:\left(l t_{14}^{2} t_{24}^{2}-2 a t_{14}^{2} t_{24}+2 b t_{14}^{2} t_{24}-l t_{14}^{2}+4 l t_{14} t_{24}-l t_{24}^{2}-2 a t_{24}+2 b t_{24}+l\right) x_{0}^{2}+\left(2 b t_{14}^{2} t_{24}^{2}-2\right.$ $\left.b t_{14}^{2}+2 b t_{24}^{2}-2 b\right) x_{0} x_{3}+\left(-2 t_{14}^{2} t_{24}^{2}+2 t_{14}^{2}-2 t_{24}^{2}+2\right) x_{0} y_{1}+\left(4 t_{14}^{2} t_{24}+4 t_{24}\right) x_{0} y_{2}+\left(l t_{14}^{2} t_{24}^{2}\right.$ $\left.-2 a t_{14}^{2} t_{24}-2 b t_{14}^{2} t_{24}-l t_{14}^{2}+4 l t_{14} t_{24}-l t_{24}^{2}-2 a t_{24}-2 b t_{24}+l\right) x_{1}^{2}+\left(-2 b t_{14}^{2} t_{24}^{2}+2 b t_{14}^{2}-\right.$ $\left.2 b t_{24}^{2}+2 b\right) x_{1} x_{2}+\left(2 t_{14}^{2} t_{24}^{2}-2 t_{14}^{2}+2 t_{24}^{2}-2\right) x_{1} y_{0}-\left(4 t_{14}^{2} t_{24}+4 t_{24}\right) x_{1} y_{3}+\left(l t_{14}^{2} t_{24}^{2}-2 a t_{14}^{2}\right.$ $\left.t_{24}+2 b t_{14}^{2} t_{24}-l t_{14}^{2}+4 l t_{14} t_{24}-l t_{24}^{2}-2 a t_{24}+2 b t_{24}+l\right) x_{2}^{2}+\left(-4 t_{14}^{2} t_{24}-4 t_{24}\right) x_{2} y_{0}+(-2$ $\left.t_{14}^{2} t_{24}^{2}+2 t_{14}^{2}-2 t_{24}^{2}+2\right) x_{2} y_{3}+\left(l t_{14}^{2} t_{24}^{2}-2 a t_{14}^{2} t_{24}-2 b t_{14}^{2} t_{24}-l t_{14}^{2}+4 l t_{14} t_{24}-l t_{24}^{2}-2 a t_{24}\right.$ $\left.-2 b t_{24}+l\right) x_{3}^{2}+\left(4 t_{14}^{2} t_{24}+4 t_{24}\right) x_{3} y_{1}+\left(2 t_{14}^{2} t_{24}^{2}-2 t_{14}^{2}+2 t_{24}^{2}-2\right) x_{3} y_{2}=0$

The Study equation in Eq. (7) is added since all solutions have to be within the Study quadric, i.e.: $g_{13}: x_{0} y_{0}+x_{1} y_{1}+x_{2} y_{2}+x_{3} y_{3}=0$. To exclude the exceptional generator $\left(x_{0}=\right.$ $x_{1}=x_{2}=x_{3}=0$ ), we add the following normalization equation: $g_{14}: x_{0}^{2}+x_{1}^{2}+x_{2}^{2}+x_{3}^{2}-1=0$. It assures that there is no point of the exceptional generators appears as a solution.

\section{Operation modes}

The 4-RUU PM is an over-constrained mechanism [28, 29], therefore it can be decomposed into two iso-constrained 2-RUU PM as shown in Fig. 4. The printed model of the 4-RUU PM presented in Fig. 2, can also be decomposed into 2-RUU PM as shown in Fig. 5. The first mechanism consists of the 1 st and the 3rd limbs, hence it is named the $2-\operatorname{RUU}_{(I)}$ PM. The second mechanism consists of the 2 nd and the 4 th limbs, hence it is named the 2-RUU $U_{(I I)} \mathrm{PM}$. 


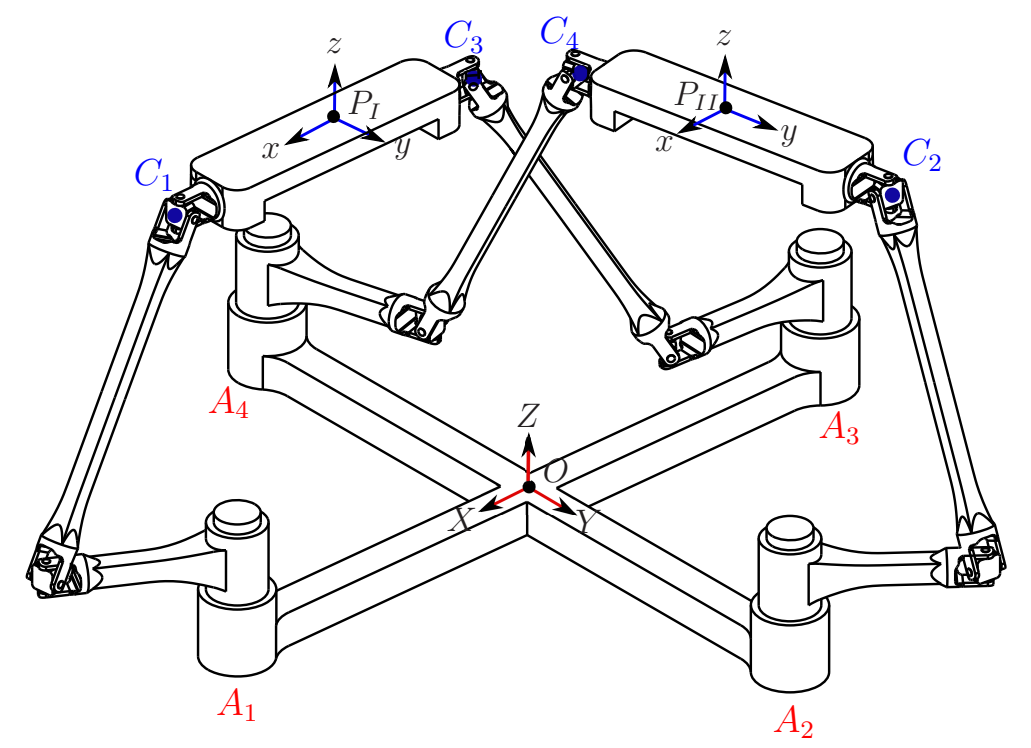

Figure 4: The 4-RUU PM decomposed into two 2-RUU PM.

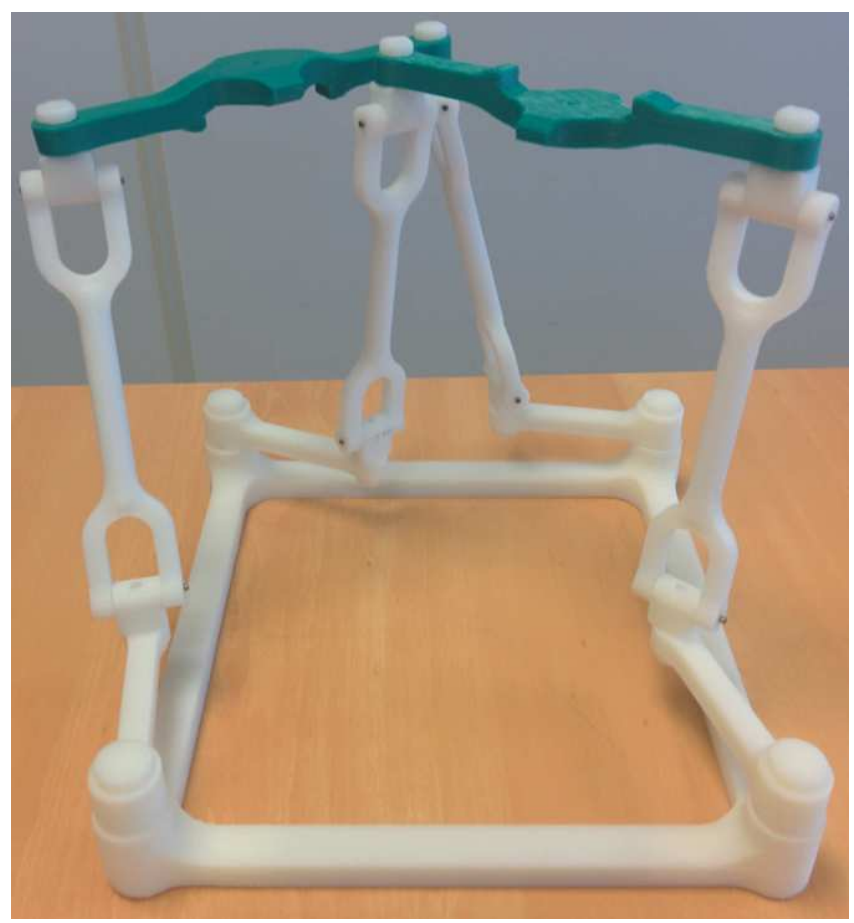

Figure 5: Printed model of 4-RUU PM decomposed into two 2-RUU PM. 
The moving platforms of both mechanisms move independently. When the moving frames of both mechanisms are coincident (accordingly $P_{I} \equiv P_{I I}$ ), we obtain the 4-RUU PM. As a consequence, the operation modes of the 4-RUU PM are determined by the linear combination of the results of primary decomposition of the $2-\mathrm{RUU}_{(I)}$ and the $2-\mathrm{RUU}\left(\mathrm{U}_{(I)} \mathrm{PM}\right.$, as presented in the following. The operation modes and the self-motions of the 2-RUU PM are presented in more detail in [31].

\subsection{The 2-RUU $(I)$ PM}

In the 2-RUU $(I)$ PM, the first and the second R-joints in each limb are actuated. The design parameters are assigned as $a=2, b=1, l=1, r=2$ (in units that need nod be specified). The set of eight constraint equations is written as a polynomial ideal with variables $\left\{x_{0}, x_{1}, x_{2}, x_{3}, y_{0}, y_{1}\right.$, $\left.y_{2}, y_{3}\right\}$ over the coefficient ring $\mathbb{C}\left[t_{11}, t_{13}, t_{21}, t_{23}\right]$, defined as: $\mathcal{I}_{(I)}=\left\langle g_{1}, g_{3}, g_{5}, g_{7}, g_{9}, g_{11}, g_{13}, g_{14}\right\rangle$. At this point, the following ideal is examined: $\mathcal{J}_{(I)}=\left\langle g_{1}, g_{3}, g_{13}\right\rangle$.

The primary decomposition is computed to verify if the ideal $\mathcal{J}_{(I)}$ is the intersection of several smaller ideals. Indeed, the ideal $\mathcal{J}_{(I)}$ is decomposed into three components as: $\mathcal{J}_{(I)}=\bigcap_{k=1}^{3} \mathcal{J}_{k(I)}$, with the results of primary decomposition:

$$
\begin{aligned}
& \mathcal{J}_{1(I)}=\left\langle x_{0}, x_{3}, x_{1} y_{1}+x_{2} y_{2}\right\rangle \\
& \mathcal{J}_{2(I)}=\left\langle x_{1}, x_{2}, x_{0} y_{0}+x_{3} y_{3}\right\rangle \\
& \mathcal{J}_{3(I)}=\left\langle\left(t_{11}^{2} t_{13}+2 t_{11} t_{13}^{2}+t_{11}+2 t_{13}\right) x_{3} y_{0}+\left(t_{11}^{2} t_{13}^{2}-1\right) x_{1} y_{1}+\ldots\right\rangle
\end{aligned}
$$

Accordingly, the 2-RUU $(I)$ PM under study has three operation modes. The computation of the Hilbert dimension of ideal $\mathcal{J}_{k(I)}$ with $t_{11}, t_{13}, t_{21}, t_{23}$ treated as variables shows that: $\operatorname{dim}\left(\mathcal{J}_{k(I)}\right)=4(k=1, \ldots, 3)$. To complete the analysis, the remaining equations are added by writing:

$$
\mathcal{K}_{k(I)}: \mathcal{J}_{k(I)} \cup\left\langle g_{5}, g_{7}, g_{9}, g_{11}, g_{14}\right\rangle, \quad k=1, \ldots, 3
$$

It follows that the 2-RUU $(I)$ PM has three 4-dof operation modes. This type of manipulator is called invariable-dof PM in [14]. Each system $\mathcal{K}_{k(I)}$ corresponds to a specific operation mode that will be discussed in the following.

\subsubsection{System $\mathcal{K}_{1(I)}:$ 1st Schönflies mode}

In this operation mode, the moving platform is reversed about an axis parallel to the $X Y$-plane of $\Sigma_{0}$ by 180 degrees from the "identity condition". The identity condition is when the moving frame and the fixed frame are coincident, i.e. $\Sigma_{1} \equiv \Sigma_{0}$ and the transformation matrix is an 
identity matrix. The condition $x_{0}=0, x_{3}=0, x_{1} y_{1}+x_{2} y_{2}=0$ are valid for all poses and are substituted into the transformation matrix $\mathbf{M}$, such that:

$$
\mathbf{M}_{1(I)}=\left(\begin{array}{cccc}
1 & 0 & 0 & \\
2\left(x_{1} y_{0}-x_{2} y_{3}\right) & x_{1}^{2}-x_{2}^{2} & 2 x_{1} x_{2} & 0 \\
2\left(x_{1} y_{3}+x_{2} y_{0}\right) & 2 x_{1} x_{2} & -x_{1}^{2}+x_{2}^{2} & 0 \\
-\frac{2 y_{2}}{x_{1}} & 0 & 0 & -1
\end{array}\right)
$$

From the transformation matrix $\mathbf{M}_{1(I)}$, it can be seen that the 2-RUU $U_{(I)}$ PM has 3-dof translational motions, which are parametrized by $y_{0}, y_{2}, y_{3}$ and 1-dof rotational motion, which is parametrized by $x_{1}, x_{2}$ in connection with $x_{1}^{2}+x_{2}^{2}-1=0$ [8]. The $z$-axis of frame $\Sigma_{1}$ attached to the moving platform is always pointing downward in this operation mode and the moving platform remains parallel to the base.

\subsubsection{System $\mathcal{K}_{2(I)}$ : 2nd Schönflies mode}

In this operation mode, the condition $x_{1}=0, x_{2}=0, x_{0} y_{0}+x_{3} y_{3}=0$ are valid for all poses. The transformation matrix in this operation mode is written as:

$$
\mathbf{M}_{2(I)}=\left(\begin{array}{cccc}
1 & 0 & 0 & 0 \\
-2\left(x_{0} y_{1}-x_{3} y_{2}\right) & x_{0}^{2}-x_{3}^{2} & -2 x_{0} x_{3} & 0 \\
-2\left(x_{0} y_{2}+x_{3} y_{1}\right) & 2 x_{0} x_{3} & x_{0}^{2}-x_{3}^{2} & 0 \\
-\frac{2 y_{3}}{x_{0}} & 0 & 0 & 1
\end{array}\right)
$$

From the transformation matrix $\mathbf{M}_{2(I)}$, it can be seen that the 2-RUU $U_{(I)}$ PM has 3-dof translational motions, which are parametrized by $y_{1}, y_{2}, y_{3}$ and 1-dof rotational motion, which is parametrized by $x_{0}, x_{3}$ in connection with $x_{0}^{2}+x_{3}^{2}-1=0$ [8]. In this operation mode, the $z$-axis of frame $\Sigma_{1}$ attached the moving platform is always pointing upward and the moving platform remains parallel to the base.

The systems $\mathcal{K}_{1(I)}$ and $\mathcal{K}_{2(I)}$ have the same motion type, i.e. Schönflies motion, however they do not have configurations in common. It occurs since the orientation of the moving platform is not the same from one operation mode to the other. The $z$-axis of frame $\Sigma_{1}$ attached to the moving platform in system $\mathcal{K}_{1(I)}$ is always pointing downward (the moving platform is always titled by 180 degrees), while in the system $\mathcal{K}_{2(I)}$, the $z$-axis of frame $\Sigma_{1}$ attached to the moving platform is always pointing upward.

\subsubsection{System $\mathcal{K}_{3(I)}$ : Third mode}

In this operation mode, the moving platform is no longer parallel to the base. The variables $x_{3}, y_{0}, y_{1}$ can be solved linearly from the ideal $\mathcal{J}_{3(I)}$ and are shown in Eq. (18). Since solving the 
inverse kinematics of $t_{11}, t_{13}$ are quite computationally expensive, the joint variables $t_{11}, t_{13}$ are considered to be the independent parameters of this mode. Then the parameters $y_{2}, y_{3}$ can be solved in terms of $\left(x_{0}, x_{1}, x_{2}, t_{11}, t_{13}\right)$. Substituting back the parameters $y_{2}, y_{3}$ into Eq. (18), then the Study parameters $x_{3}, y_{0}, y_{1}, y_{2}, y_{3}$ are now parametrized by $\left(x_{0}, x_{1}, x_{2}, t_{11}, t_{13}\right)$. Accordingly, the 2-RUU $U_{(I)} \mathrm{PM}$ will perform two translational motions, which are parametrized by variables $t_{11}, t_{13}$ and two rotational motions, which are parametrized by variables $x_{0}, x_{1}, x_{2}$ in connection with the normalization equation $g_{14}$.

$$
\begin{aligned}
x_{3(I)}= & \frac{\left(t_{11}^{2} t_{13}^{2} x_{1}-t_{11}^{2} t_{13} x_{2}+t_{11} t_{13}^{2} x_{2}+2 t_{13}^{2} x_{1}+t_{11} x_{2}-t_{13} x_{2}+x_{1}\right) x_{0}}{\left(3 t_{11}^{2} t_{13}^{2} x_{2}+t_{11}^{2} t_{13} x_{1}-t_{11} t_{13}^{2} x_{1}+2 t_{11}^{2} x_{2}+4 t_{13}^{2} x_{2}-t_{11} x_{1}+t_{13} x_{1}+3 x_{2}\right)} \\
y_{0(I)}= & -\frac{1}{3 t_{11}^{2} t_{13}^{2} x_{2}+t_{11}^{2} t_{13} x_{1}-t_{11} t_{13}^{2} x_{1}+2 t_{11}^{2} x_{2}+4 t_{13}^{2} x_{2}-t_{11} x_{1}+t_{13} x_{1}+3 x_{2}}\left(t_{11}^{2} t_{13}^{2} x_{1} x_{2}+\right. \\
& t_{11}^{2} t_{13}^{2} x_{1} y_{3}-t_{11}^{2} t_{13} x_{2}^{2}-t_{11}^{2} t_{13} x_{2} y_{3}-t_{11} t_{13}^{2} x_{1}^{2}-2 t_{11} t_{13}^{2} x_{2}^{2}+t_{11} t_{13}^{2} x_{2} y_{3}+2 t_{13}^{2} x_{1} y_{3}-t_{11} \\
& \left.x_{2}^{2}+t_{11} x_{2} y_{3}-t_{13} x_{1}^{2}-2 t_{13} x_{2}^{2}-t_{13} x_{2} y_{3}-x_{1} x_{2}+x_{1} y_{3}\right) \\
y_{1(I)}= & \frac{1}{\left(3 t_{11}^{2} t_{13}^{2} x_{2}+t_{11}^{2} t_{13} x_{1}-t_{11} t_{13}^{2} x_{1}+2 t_{11}^{2} x_{2}+4 t_{13}^{2} x_{2}-t_{11} x_{1}+t_{13} x_{1}+3 x_{2}\right) x_{1}}\left(t_{11}^{2} t_{13}^{2} x_{0}\right. \\
& x_{1} x_{2}-3 t_{11}^{2} t_{13}^{2} x_{2}^{2} y_{2}-t_{11}^{2} t_{13} x_{0} x_{2}^{2}-t_{11}^{2} t_{13} x_{1} x_{2} y_{2}-t_{11} t_{13}^{2} x_{0} x_{1}^{2}-2 t_{11} t_{13}^{2} x_{0} x_{2}^{2}+t_{11} t_{13}^{2} x_{1} \\
& x_{2} y_{2}-2 t_{11}^{2} x_{2}^{2} y_{2}-4 t_{13}^{2} x_{2}^{2} y_{2}-t_{11} x_{0} x_{2}^{2}+t_{11} x_{1} x_{2} y_{2}-t_{13} x_{0} x_{1}^{2}-2 t_{13} x_{0} x_{2}^{2}-t_{13} x_{1} x_{2} y_{2} \\
& \left.-x_{0} x_{1} x_{2}-3 x_{2}^{2} y_{2}\right)
\end{aligned}
$$

Under this operation mode, the joint angles $t_{21}$ and $t_{23}$ can be computed from the equations $g_{9}, g_{11}$. It turns out that no matter the value of the first actuated joints $\left(t_{11}, t_{13}\right)$ in each limb, these equations vanish for two real solutions, namely (1.) $t_{21}=-\frac{1}{t_{23}}\left(\theta_{21}=\pi+\theta_{23}\right)$ and (2.) $t_{21}=t_{23}\left(\theta_{21}=\theta_{23}\right)$. As a consequence, in this operation mode, the links $B_{i} C_{i}(i=1,3)$ from both limbs are always parallel to the same plane and the axes $\mathbf{s}_{i}(i=1,3)$ from both limbs are always parallel too.

\subsection{The 2-RUU $(I I)$ PM}

In the 2-RUU $(I I)$ PM, the first and the second R-joints in each limb are also actuated. The design parameters are assigned with the same values as $a=2, b=1, l=1, r=2$ (in units that need not be specified). The set of eight constraint equations is written as a polynomial ideal with variables $\left\{x_{0}, x_{1}, x_{2}, x_{3}, y_{0}, y_{1}, y_{2}, y_{3}\right\}$ over the coefficient ring $\mathbb{C}\left[t_{12}, t_{14}, t_{22}, t_{24}\right]$, defined as: $\mathcal{I}_{(I I)}=\left\langle g_{2}, g_{4}, g_{6}, g_{8}, g_{10}, g_{12}, g_{13}, g_{14}\right\rangle$. At this point, the following ideal is examined: $\mathcal{J}_{(I I)}=$ $\left\langle g_{2}, g_{4}, g_{13}\right\rangle$.

The primary decomposition is computed and it turns out that the ideal $\mathcal{J}_{(I I)}$ is decomposed 
into three components as: $\mathcal{J}_{(I I)}=\bigcap_{k=1}^{3} \mathcal{J}_{k(I I)}$, with the results of primary decomposition:

$$
\begin{aligned}
\mathcal{J}_{1(I I)} & =\left\langle x_{0}, x_{3}, x_{1} y_{1}+x_{2} y_{2}\right\rangle \\
\mathcal{J}_{2(I I)} & =\left\langle x_{1}, x_{2}, x_{0} y_{0}+x_{3} y_{3}\right\rangle \\
\mathcal{J}_{3(I I)} & =\left\langle\left(3 t_{12}^{2} t_{14}^{2}-t_{12}^{2} t_{14}+t_{12} t_{14}^{2}-t_{12}+t_{14}-3\right) x_{3} y_{0}+\left(-t_{12}^{2} t_{14}^{2}+\ldots\right\rangle\right.
\end{aligned}
$$

Accordingly, the 2-RUU $(I I)$ PM under study has three operation modes. The computation of the Hilbert dimension of ideal $\mathcal{J}_{k(I I)}$ with $t_{12}, t_{14}, t_{22}, t_{24}$ treated as variables shows that: $\operatorname{dim}\left(\mathcal{J}_{k(I I)}\right)=4(k=1, \ldots, 3)$. To complete the analysis, the remaining equations are added by writing:

$$
\mathcal{K}_{k(I I)}: \mathcal{J}_{k(I I)} \cup\left\langle g_{6}, g_{8}, g_{10}, g_{12}, g_{14}\right\rangle, \quad k=1, \ldots, 3
$$

It follows that the 2-RUU $U_{(I I)} \mathrm{PM}$ has three 4-dof operation modes too. The system $\mathcal{K}_{1(I I)}$ is identical with the system $\mathcal{K}_{1(I)}$ (as explained in Section 4.1.1), in which the moving platform is titled about an axis of parallel to $X Y$-plane of $\Sigma_{0}$ by 180 degrees and it can exhibit the Schönflies motion with pure rotation about $Z$-axis. The system $\mathcal{K}_{2(I I)}$ is identical with the system $\mathcal{K}_{2(I)}$ (as explained in Section 4.1.2), where the moving platform can exhibit 3-dof independent translations and one pure rotation about $Z$-axis. In the system $\mathcal{K}_{3(I I)}$, the moving platform is no longer parallel to the base. The variables $x_{3}, y_{0}, y_{1}$ can be solved linearly from the ideal $\mathcal{J}_{3(I I)}$ and are shown in Eq. (21). Since solving the inverse kinematics of $t_{12}, t_{14}$ are quite computationally expensive, the joint variables $t_{12}, t_{14}$ are considered to be the independent parameters in this third mode. Then the parameters $y_{2}, y_{3}$ can be solved in terms of $\left(x_{0}, x_{1}, x_{2}, t_{12}, t_{14}\right)$. Substituting back the parameters $y_{2}, y_{3}$ into Eq. (21), the Study parameters $x_{3}, y_{0}, y_{1}, y_{2}, y_{3}$ are now obtained and parametrized by $\left(x_{0}, x_{1}, x_{2}, t_{12}, t_{14}\right)$. Hence the moving platform of the 2-RUU ${ }_{(I I)}$ PM will perform two translational motions which are parametrized by $t_{12}, t_{14}$ and two rotational motions which are parametrized by $x_{0}, x_{1}, x_{2}$ in connection with the normalization equation $g_{14}$.

Under the system $\mathcal{K}_{3(I I)}$, the joint angles $t_{22}$ and $t_{24}$ can be computed from the equations $g_{10}, g_{12}$. It reveals that no matter the value of the first actuated joint $\left(t_{12}, t_{14}\right)$ in each limb, these equations $\left(g_{10}, g_{12}\right)$ vanish for two real solutions, namely (1.) $t_{22}=-\frac{1}{t_{24}}\left(\theta_{22}=\pi+\theta_{24}\right)$ and (2.) $t_{22}=t_{24}\left(\theta_{22}=\theta_{24}\right)$. It means that in this operation mode, the links $B_{i} C_{i}(i=2,4)$ from both limbs are always parallel to the same plane and the axes $\mathbf{s}_{i}(i=2,4)$ from both limbs are always parallel too. 


$$
\begin{aligned}
& x_{3(I I)}=-\frac{\left(t_{12}^{2} t_{14}^{2} x_{2}-t_{12}^{2} t_{14} x_{2}+t_{12} t_{14}^{2} x_{2}-t_{12}^{2} x_{1}+t_{12}^{2} x_{2}+t_{14}^{2} x_{1}+t_{14}^{2} x_{2}+t_{12} x_{2}-t_{14} x_{2}+\right.}{3 t_{12}^{2} t_{14}^{2} x_{1}-t_{12}^{2} t_{14} x_{1}+t_{12} t_{14}^{2} x_{1}+3 t_{12}^{2} x_{1}+t_{12}^{2} x_{2}+3 t_{14}^{2} x_{1}-t_{14}^{2} x_{2}+t_{12} x_{1}-t_{14}} \\
& \frac{\left.x_{2}\right) x_{0}}{x_{1}+3 x_{1}} \\
& y_{0(I I)}=-\frac{1}{2\left(3 t_{12}^{2} t_{14}^{2} x_{1}-t_{12}^{2} t_{14} x_{1}+t_{12} t_{14}^{2} x_{1}+3 t_{12}^{2} x_{1}+t_{12}^{2} x_{2}+3 t_{14}^{2} x_{1}-t_{14}^{2} x_{2}+t_{12} x_{1}-t_{14}\right.} \\
& \overline{\left.x_{1}+3 x_{1}\right)}\left(3 t_{12}^{2} t_{14}^{2} x_{1}^{2}+t_{12}^{2} t_{14}^{2} x_{2}^{2}-2 t_{12}^{2} t_{14}^{2} x_{2} y_{3}-t_{12}^{2} t_{14} x_{1}^{2}-2 t_{12}^{2} t_{14} x_{1} x_{2}-t_{12}^{2} t_{14} x_{2}^{2}+\right. \\
& 2 t_{12}^{2} t_{14} x_{2} y_{3}+t_{12} t_{14}^{2} x_{1}^{2}-2 t_{12} t_{14}^{2} x_{1} x_{2}+t_{12} t_{14}^{2} x_{2}^{2}-2 t_{12} t_{14}^{2} x_{2} y_{3}+2 t_{12}^{2} x_{1} y_{3}-2 t_{12}^{2} x_{2} y_{3} \\
& -2 t_{14}^{2} x_{1} y_{3}-2 t_{14}^{2} x_{2} y_{3}-t_{12} x_{1}^{2}-2 t_{12} x_{1} x_{2}-t_{12} x_{2}^{2}-2 t_{12} x_{2} y_{3}+t_{14} x_{1}^{2}-2 t_{14} x_{1} x_{2}+ \\
& \left.t_{14} x_{2}^{2}+2 t_{14} x_{2} y_{3}-3 x_{1}^{2}-x_{2}^{2}-2 x_{2} y_{3}\right) \\
& y_{1(I I)}=\frac{1}{2\left(3 t_{12}^{2} t_{14}^{2} x_{1}-t_{12}^{2} t_{14} x_{1}+t_{12} t_{14}^{2} x_{1}+3 t_{12}^{2} x_{1}+t_{12}^{2} x_{2}+3 t_{14}^{2} x_{1}-t_{14}^{2} x_{2}+t_{12} x_{1}-t_{14} x_{1}\right.} \\
& \frac{}{\left.+3 x_{1}\right) x_{1}}\left(3 t_{12}^{2} t_{14}^{2} x_{0} x_{1}^{2}+t_{12}^{2} t_{14}^{2} x_{0} x_{2}^{2}-6 t_{12}^{2} t_{14}^{2} x_{1} x_{2} y_{2}-t_{12}^{2} t_{14} x_{0} x_{1}^{2}-2 t_{12}^{2} t_{14} x_{0} x_{1} x_{2}-\right. \\
& t_{12}^{2} t_{14} x_{0} x_{2}^{2}+2 t_{12}^{2} t_{14} x_{1} x_{2} y_{2}+t_{12} t_{14}^{2} x_{0} x_{1}^{2}-2 t_{12} t_{14}^{2} x_{0} x_{1} x_{2}+t_{12} t_{14}^{2} x_{0} x_{2}^{2}-2 t_{12} t_{14}^{2} x_{1} x_{2} \\
& y_{2}-6 t_{12}^{2} x_{1} x_{2} y_{2}-2 t_{12}^{2} x_{2}^{2} y_{2}-6 t_{14}^{2} x_{1} x_{2} y_{2}+2 t_{14}^{2} x_{2}^{2} y_{2}-t_{12} x_{0} x_{1}^{2}-2 t_{12} x_{0} x_{1} x_{2}-t_{12} x_{0} \\
& x_{2}^{2}-2 t_{12} x_{1} x_{2} y_{2}+t_{14} x_{0} x_{1}^{2}-2 t_{14} x_{0} x_{1} x_{2}+t_{14} x_{0} x_{2}^{2}+2 t_{14} x_{1} x_{2} y_{2}-3 x_{0} x_{1}^{2}-x_{0} x_{2}^{2}- \\
& \left.6 x_{1} x_{2} y_{2}\right)
\end{aligned}
$$

\subsection{The 4-RUU PM}

In the 4-RUU PM, the first R-joint in each limb is actuated. The design parameters are assigned with the same values as $a=2, b=1, l=1, r=2$. The set of ten constraint equations is written as a polynomial ideal with variables $\left\{x_{0}, x_{1}, x_{2}, x_{3}, y_{0}, y_{1}, y_{2}, y_{3}\right\}$ over the coefficient ring $\mathbb{C}\left[t_{11}, t_{12}, t_{13}, t_{14}\right]$, defined as: $\mathcal{I}=\left\langle g_{1}, g_{2}, g_{3}, g_{4}, g_{5}, g_{6}, g_{7}, g_{8}, g_{13}, g_{14}\right\rangle$.

At this point, the following ideal is examined: $\mathcal{J}=\left\langle g_{1}, g_{2}, g_{3}, g_{4}, g_{13}\right\rangle$. Since the 4-RUU PM can be assembled by the $2-\mathrm{RUU}_{(I)}$ and $2-\mathrm{RUU}_{(I I)} \mathrm{PM}$, the ideal $\mathcal{J}$ can be written as the linear combination of the results of primary decomposition from the $2-\mathrm{RUU}_{(I)}$ and $2-\mathrm{RUU}_{(I I)}$ PM. It is noteworthy that the first and second components of the 2-RUU $U_{(I)}$ and $2-\mathrm{RUU}_{(I I)} \mathrm{PM}$ are identical, so that $\mathcal{J}_{1(I)}=\mathcal{J}_{1(I I)}$ and $\mathcal{J}_{2(I)}=\mathcal{J}_{2(I I)}$.

$$
\mathcal{J}=\bigcap_{k=1}^{3} \mathcal{J}_{k}
$$

with: 


$$
\begin{aligned}
\mathcal{J}_{1}=\mathcal{J}_{1(I)} \cup \mathcal{J}_{1(I I)}= & \left\langle x_{0}, x_{3}, x_{1} y_{1}+x_{2} y_{2}\right\rangle \\
\mathcal{J}_{2}=\mathcal{J}_{2(I)} \cup \mathcal{J}_{2(I I)}= & \left\langle x_{1}, x_{2}, x_{0} y_{0}+x_{3} y_{3}\right\rangle \\
\mathcal{J}_{3}=\mathcal{J}_{3(I)} \cup \mathcal{J}_{3(I I)}= & \left\langle\left(t_{11}^{2} t_{13}+2 t_{11} t_{13}^{2}+t_{11}+2 t_{13}\right) x_{3} y_{0}+\left(t_{11}^{2} t_{13}^{2}-1\right) x_{1} y_{1}+\ldots,\right. \\
& \left(3 t_{12}^{2} t_{14}^{2}-t_{12}^{2} t_{14}+t_{12} t_{14}^{2}-t_{12}+t_{14}-3\right) x_{3} y_{0}+\left(-t_{12}^{2} t_{14}^{2}+\ldots\right\rangle
\end{aligned}
$$

As a consequence, the 4-RUU PM has four operation modes. To complete the analysis, the remaining equations are added by writing:

$$
\mathcal{K}_{k}: \mathcal{J}_{k} \cup\left\langle g_{5}, g_{6}, g_{7}, g_{8}, g_{14}\right\rangle, \quad k=1, \ldots, 3
$$

The systems $\mathcal{K}_{1}$ and $\mathcal{K}_{2}$ are 4-dof operation modes, which correspond to the 1 st Schönflies mode and the 2nd Schönflies mode, as explained in Sections 4.1.1 and 4.1.2, respectively. However, the characterization of the system $\mathcal{K}_{3}$ needs to be discussed further, as presented hereafter.

\subsubsection{System $\mathcal{K}_{3}$ : Third mode}

The third mode of the 4-RUU PM is characterized by the system $\mathcal{K}_{3}$. In this mode, the primary decomposition leads to the ideal $\mathcal{J}_{3}$ and all polynomial equations in this ideal should vanish simultaneously. Hence the variables $x_{3}, y_{0}, y_{1}, y_{2}, y_{3}$ can be obtained in cascade from the ideal $\mathcal{J}_{3}$, such that:

$$
\begin{gathered}
x_{3}=\begin{array}{c}
-x_{0} x_{2}\left(t_{1}^{2} t_{2}^{2} t_{3}^{2} t_{4}^{2}-t_{1}^{2} t_{2}^{2} t_{3}^{2} t_{4}+t_{1}^{2} t_{2} t_{3}^{2} t_{4}^{2}+2 t_{2}^{2} t_{3}^{2} t_{4}^{2}-t_{1}^{2} t_{2} t_{3}^{2}+t_{1}^{2} t_{3}^{2} t_{4}+2 t_{1} t_{2}^{2} t_{3}^{2}-2 t_{1} t_{3}^{2} t_{4}^{2}-2 t_{2}^{2} t_{3}^{2}\right. \\
t_{4}+2 t_{2} t_{3}^{2} t_{4}^{2}-t_{1}^{2} t_{3}^{2}+t_{2}^{2} t_{4}^{2}+2 t_{2}^{2} t_{3}-t_{2}^{2} t_{4}-2 t_{2} t_{3}^{2}+t_{2} t_{4}^{2}+2 t_{3}^{2} t_{4}-2 t_{3} t_{4}^{2}-2 t_{3}^{2}-t_{2}+t_{4}-\ldots \\
y_{0}=-x_{2}\left(t_{2}^{2} t_{4}^{2}-t_{2}^{2} t_{4}+t_{2} t_{4}^{2}-t_{2}+t_{4}-1\right) / 2\left(t_{2}^{2}-t_{4}^{2}\right) \\
y_{1}=t_{3} x_{0} x_{2}\left(t_{1} t_{2}^{2} t_{3} t_{4}^{2}-t_{1} t_{2}^{2} t_{3} t_{4}+t_{1} t_{2} t_{3} t_{4}^{2}+t_{2}^{2} t_{4}^{2}-t_{1} t_{2} t_{3}+t_{1} t_{3} t_{4}-t_{2}^{2} t_{4}+t_{2} t_{4}^{2}-t_{1} t_{3}-t_{2}+t_{4}-\right. \\
1) /\left(3 t_{1}^{2} t_{2}^{2} t_{3}^{2} t_{4}^{2} x_{1}-t_{1}^{2} t_{2}^{2} t_{3}^{2} t_{4} x_{1}+t_{1}^{2} t_{2} t_{3}^{2} t_{4}^{2} x_{1}-2 t_{1}^{2} t_{2}^{2} t_{3}^{2} x_{2}+2 t_{1}^{2} t_{3}^{2} t_{4}^{2} x_{2}+6 t_{2}^{2} t_{3}^{2} t_{4}^{2} x_{1}-t_{1}^{2} t_{2} t_{3}^{2} x_{1} \ldots\right. \\
y_{2}=-\left(t_{1}^{2} t_{2}^{2} t_{3}^{2} t_{4}^{2} x_{2}-t_{1}^{2} t_{2}^{2} t_{3}^{2} t_{4} x_{2}+t_{1}^{2} t_{2} t_{3}^{2} t_{4}^{2} x_{2}+3 t_{1} t_{2}^{2} t_{3}^{2} t_{4}^{2} x_{1}-t_{1} t_{2}^{2} t_{3}^{2} t_{4} x_{1}+t_{1} t_{2} t_{3}^{2} t_{4}^{2} x_{1}-t_{1}^{2} t_{2} t_{3}^{2} x_{2}+\right. \\
t_{1}^{2} t_{3}^{2} t_{4} x_{2}+3 t_{2}^{2} t_{3} t_{4}^{2} x_{1}-t_{1}^{2} t_{3}^{2} x_{2}-t_{1} t_{2} t_{3}^{2} x_{1}+t_{1} t_{3}^{2} t_{4} x_{1}-t_{2}^{2} t_{3} t_{4} x_{1}-t_{2}^{2} t_{4}^{2} x_{2}+t_{2} t_{3} t_{4}^{2} x_{1}-3 t_{1} \ldots \\
y_{3}=-\left(3 t_{2}^{2} t_{4}^{2}-t_{2}^{2} t_{4}+t_{2} t_{4}^{2}-t_{2}+t_{4}-3\right) x_{1} / 2\left(t_{2}^{2}-t_{4}^{2}\right)
\end{array} \\
(25 \mathrm{~d})
\end{gathered}
$$

Not all polynomial in the ideal $\mathcal{J}_{3}$ vanishes and it remains two polynomial equations, as follows: 


$$
\begin{aligned}
& f_{1}: 3 t_{1}^{2} t_{2}^{2} t_{3}^{2} t_{4}^{2} x_{1}^{2}+3 t_{1}^{2} t_{2}^{2} t_{3}^{2} t_{4}^{2} x_{2}^{2}-t_{1}^{2} t_{2}^{2} t_{3}^{2} t_{4} x_{1}^{2}-3 t_{1}^{2} t_{2}^{2} t_{3}^{2} t_{4} x_{2}^{2}-2 t_{1}^{2} t_{2}^{2} t_{3} t_{4}^{2} x_{1} x_{2}+t_{1}^{2} t_{2} t_{3}^{2} t_{4}^{2} x_{1}^{2}+\ldots=0 \\
& f_{2}: 3 t_{1}^{2} t_{2}^{2} t_{3}^{2} t_{4}^{2} x_{1}^{2}+3 t_{1}^{2} t_{2}^{2} t_{3}^{2} t_{4}^{2} x_{2}^{2}-t_{1}^{2} t_{2}^{2} t_{3}^{2} t_{4} x_{1}^{2}-2 t_{1}^{2} t_{2}^{2} t_{3}^{2} t_{4} x_{1} x_{2}-3 t_{1}^{2} t_{2}^{2} t_{3}^{2} t_{4} x_{2}^{2}+t_{1}^{2} t_{2} t_{3}^{2} t_{4}^{2} x_{1}^{2}-\ldots=0
\end{aligned}
$$

As two iso-constrained 2-RUU PM are assembled to be the 4-RUU PM by combining the results of primary decomposition into $\mathcal{J}_{3}$, one of the 2-RUU PM is dependent to another. Accordingly, one of the 2-RUU PM can be selected to represent the third mode of the 4-RUU PM. The new ideals are defined corresponding to the two 2-RUU parallel manipulators as follows:

$$
\begin{array}{ll}
\mathcal{L}_{I}: & \left\langle g_{5}, g_{7}, g_{14}, f_{1}, f_{2}\right\rangle \\
\mathcal{L}_{I I}: & \left\langle g_{6}, g_{8}, g_{14}, f_{1}, f_{2}\right\rangle
\end{array}
$$

Both ideals in Eq. (27) are solved separately to show that they lead to the same results. The variables $x_{3}, y_{0}, y_{1}, y_{2}, y_{3}$ obtained in Eq. (25) are then substituted into ideals $\mathcal{L}_{I}, \mathcal{L}_{I I}$ in Eq. (27). The variable $x_{0}$ can be solved from $g_{14}$ and the equations $\left\langle g_{5}, g_{6}, g_{7}, g_{8}\right\rangle$ split into two factors. The first factors of these equations have the same mathematical expression and lead to the 1-dof self-motion, which will be discussed further in Section 5.3.

The second factors are analysed thereafter. The variable $x_{1}$ is solved from each ideal and yields two equations in terms of $\left(x_{2}, t_{11}, t_{12}, t_{13}, t_{14}\right)$ and one equation in terms of the actuated joint angles only $\left(t_{11}, t_{12}, t_{13}, t_{14}\right)$, which should be fulfilled by the manipulator as:

$$
\begin{aligned}
& 3 t_{11}^{4} t_{12}^{4} t_{13}^{4} t_{14}^{2}-6 t_{11}^{4} t_{12}^{4} t_{13}^{3} t_{14}^{3}+3 t_{11}^{4} t_{12}^{4} t_{13}^{2} t_{14}^{4}+6 t_{11}^{4} t_{12}^{3} t_{13}^{4} t_{14}^{3}-6 t_{11}^{4} t_{12}^{3} t_{13}^{3} t_{14}^{4}+3 t_{11}^{4} t_{12}^{2} t_{13}^{4} t_{14}^{4}-6 \\
& t_{11}^{3} t_{12}^{4} t_{13}^{4} t_{14}^{3}+6 t_{11}^{3} t_{12}^{4} t_{13}^{3} t_{14}^{4}-6 t_{11}^{3} t_{12}^{3} t_{13}^{4} t_{14}^{4}+3 t_{11}^{2} t_{12}^{4} t_{13}^{4} t_{14}^{4}+3 t_{11}^{4} t_{12}^{4} t_{13}^{4}-2 t_{11}^{4} t_{12}^{4} t_{13}^{3} t_{14}-\ldots=0
\end{aligned}
$$

To avoid computational burden, some values are assigned to two actuated joint angles as $t_{11}=-\frac{9}{10}, t_{12}=-2$ and variables $x_{2}$ can be solved. Eventually, one equation in terms of $t_{13}, t_{14}$ remains in ideals $\mathcal{L}_{I}, \mathcal{L}_{I I}$ as shown in Eq. (29). Back substitution into Eqs. (25)-(28), all Study parameters can be solved and one of the manipulator poses can be obtained as shown in Fig. 6 with $\theta_{11}=-84^{\circ}, \theta_{12}=126.934^{\circ}, \theta_{13}=64.243^{\circ}, \theta_{14}=56.901^{\circ}$.

$$
\begin{aligned}
& \left(t_{4}-2\right)\left(t_{4}+2\right)\left(51597 t_{3}^{2} t_{4}^{4}-18144 t_{3}^{2} t_{4}^{3}-2520 t_{3} t_{4}^{4}-27855 t_{3}^{2} t_{4}^{2}+1086 t_{3} t_{4}^{3}+29600 t_{4}^{4}+4932 t_{3}^{2} t_{4}\right. \\
& \left.+21642 t_{3} t_{4}^{2}-13800 t_{4}^{3}+4680 t_{3}^{2}-4344 t_{3} t_{4}-13771 t_{4}^{2}-5748 t_{3}+3846 t_{4}+2801\right)\left(742 t_{3}^{2} t_{4}^{2}-843\right. \\
& \left.t_{3}^{2} t_{4}-200 t_{3} t_{4}^{2}-439 t_{3}^{2}+200 t_{4}^{2}+800 t_{3}-300 t_{4}+100\right)\left(2990 t_{3}^{2} t_{4}^{2}-843 t_{3}^{2} t_{4}-200 t_{3} t_{4}^{2}-1001 t_{3}^{2}+\right. \\
& \left.1000 t_{4}^{2}+800 t_{3}-300 t_{4}-100\right)=0
\end{aligned}
$$


Noticeably, the third mode of the 4-RUU PM is a 2-dof operation mode since two input joint angles are sufficient to define the pose of the manipulator. This operation mode was referred to coupled motion in $[28,29]$. Since the system $\mathcal{K}_{3}$ is a lower dimension operation mode, namely 2-dof, this type of manipulator is called variable-dof PM in [14].

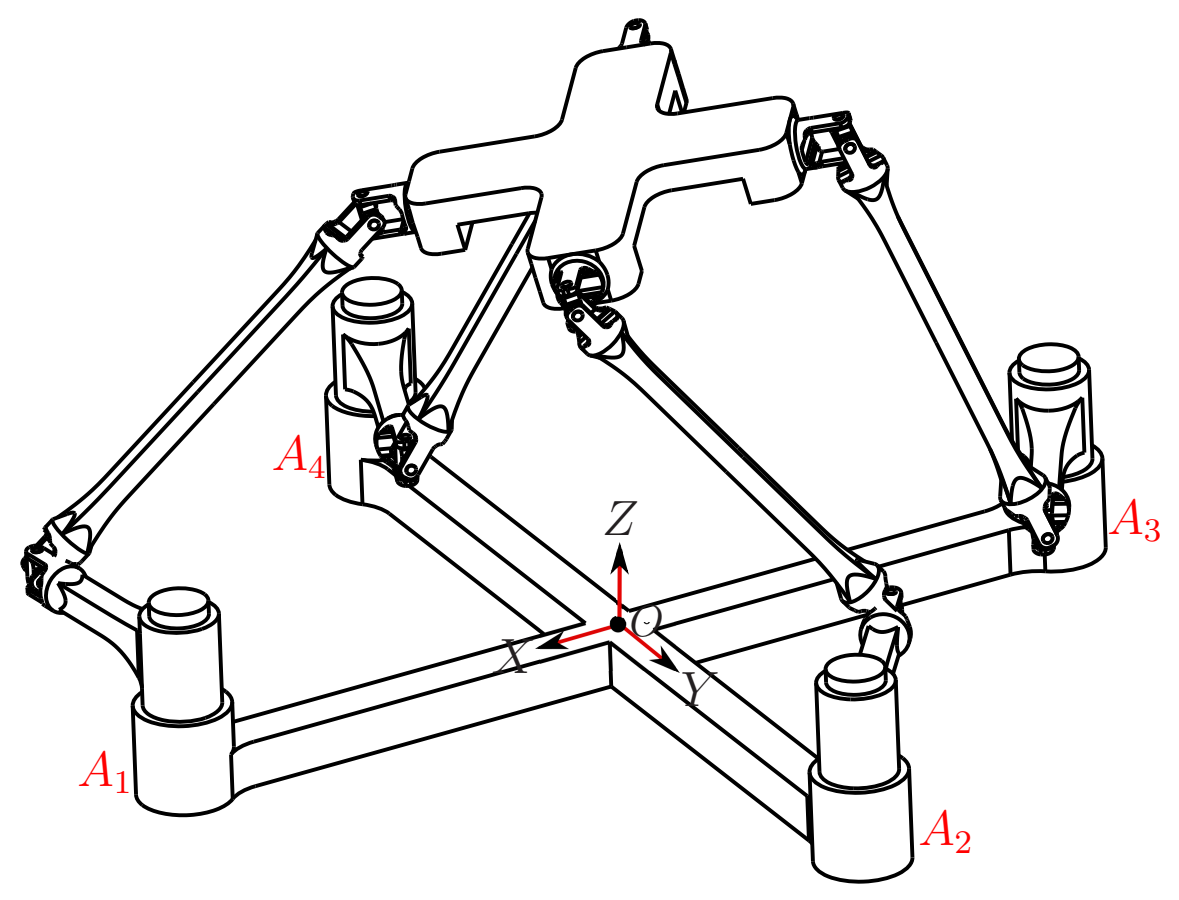

Figure 6: The third mode $\mathcal{K}_{3}$.

\section{$5 \quad$ Singularities and Self-motions}

The 4-RUU PM reaches a singularity condition when the determinant of its Jacobian matrix vanishes. The Jacobian matrix is the matrix of all first order partial derivative of the constraint equations with respect to the Study parameters. Since the 4-RUU PM has more than one operation mode, the singular configurations can be classified into two different types, i.e. the singularity configurations that belong to a single operation mode and the singularity configurations that belong to more than one operation mode. The common configurations than belong to more than one operation mode allow the 4-RUU PM to switch from one operation mode to another operation mode, which will be discussed in Section 6 .

The singular poses are examined by taking the Jacobian matrix from each system of polynomial and computing its determinant. From practical point of view, the singularity surface is desirable also in the joint space. Hence the expression of the Jacobian determinant is added 
into each system $\mathcal{K}_{k}$ and all Study parameters are eliminated to obtain a single polynomial in the joint variables $t_{11}, t_{12}, t_{13}, t_{14}$. The detail analysis of the singularity conditions in each operation mode is discussed in the following.

\subsection{Singularities in 1st Schönflies mode $\left(\mathcal{K}_{1}\right)$}

The determinant of the Jacobian matrix is computed in the system $\mathcal{K}_{1}$, which consists of five constraint equations over five variables $\left(x_{1}, x_{2}, y_{0}, y_{2}, y_{3}\right)$. Hence the $5 \times 5$ Jacobian matrix can be obtained. The factorization of the determinant of this Jacobian matrix $\mathcal{S}_{1}: \operatorname{det}\left(\mathbf{J}_{1}\right)=0$ yields three factors. The inspection of the first factor shows the singularity configurations that lie in the intersection with the system $\mathcal{K}_{2}$. However, this factor is neglected since the systems $\mathcal{K}_{1}$ and $\mathcal{K}_{2}$ do not have configurations in common, i.e. $x_{0}, x_{1}, x_{2}, x_{3}$ can never vanish simultaneously.

The second factor is $y_{2}=0$, when the moving platform is coplanar to the base, the 4-RUU $\mathrm{PM}$ is always in a singular configuration. Eventually, the third factor of $\mathcal{S}_{1}: \operatorname{det}\left(\mathbf{J}_{1}\right)=0$ is analysed. This factor is added to the system $\mathcal{K}_{1}$ and the remaining five Study parameters are eliminated. Due to the heavy elimination process, the actuated joint angles are assigned as $t_{11}=-2, t_{12}=-1$, and $t_{13}=1 / 2$. The elimination yields a univariate polynomial of degree 14 in $t_{14}$ as:

$$
\begin{aligned}
& 2028993300 t_{4}^{1} 4-16353282060 t_{4}^{1} 3+62397559157 t_{4}^{1} 2-149262975886 t_{4}^{1} 1+248592910386 t_{4}^{1} 0- \\
& 303624070978 t_{4}^{9}+279023850507 t_{4}^{8}-193904900892 t_{4}^{7}+100410321408 t_{4}^{6}-37073611168 t_{4}^{5}+ \\
& 8730261899 t_{4}^{4}-928156246 t_{4}^{3}-54729862 t_{4}^{2}+18830830 t_{4}-1000395=0
\end{aligned}
$$

Let us consider one singularity configuration of the 4-RUU PM in the 1st Schönflies mode by solving Eq. (30) for $t_{14}=1$. Hence the direct kinematics of at least one singularity configuration can be obtained with $\theta_{11}=126.934^{\circ}, \theta_{12}=-90^{\circ}, \theta_{13}=53.157^{\circ}, \theta_{14}=90^{\circ}$ and it is shown in Fig. 7.

\subsection{Singularities and Self-motions in 2nd Schönflies mode $\left(\mathcal{K}_{2}\right)$}

The determinant of the Jacobian matrix is computed in the system $\mathcal{K}_{2}$, which consists of five constraint equations over five variables. Therefore the $5 \times 5$ Jacobian matrix can be obtained. The determinant of this Jacobian matrix $\mathcal{S}_{2}: \operatorname{det}\left(\mathbf{J}_{2}\right)=0$ consists of three factors too. The investigation of the first factor gives the condition in which the mechanism is in the intersection between the systems $\mathcal{K}_{1}$ and $\mathcal{K}_{2}$. As explained in Section 5.1, this factor is removed. 


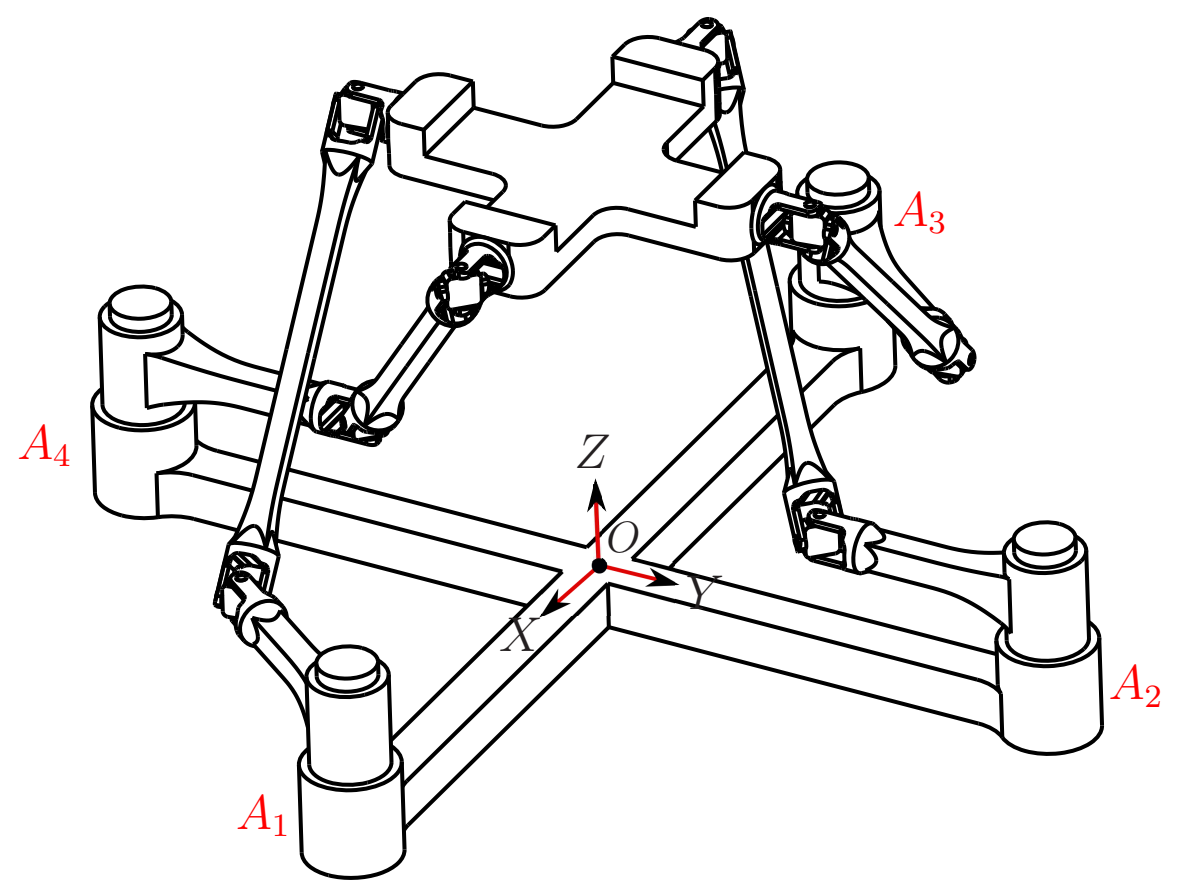

Figure 7: Singularity pose in the 1st Schönflies mode $\mathcal{K}_{1}$.

The second factor is $y_{3}=0$, when the moving platform is coplanar to the base, the 4-RUU $\mathrm{PM}$ is always in a singular configuration. Finally, the last factor of $\mathcal{S}_{2}: \operatorname{det}\left(\mathbf{J}_{2}\right)=0$ is analysed. Due to the heavy elimination process, the actuated joint angles are assigned as $t_{11}=0, t_{12}=1$, and $t_{13}=0$. The elimination gives a univariate polynomial of degree 18 in $t_{14}$ as:

$$
\begin{aligned}
& 99544625 t_{14}^{18}-1042686200 t_{14}^{17}+4293155895 t_{14}^{16}-9293913184 t_{14}^{15}+10513736564 t_{14}^{14}-175591 \\
& 6864 t_{14}^{13}-14239053636 t_{14}^{12}+24856530336 t_{14}^{11}-20314694418 t_{14}^{10}+4683758224 t_{14}^{9}+92888105 \\
& 78 t_{14}^{8}-13708185120 t_{14}^{7}+10456187332 t_{14}^{6}-5370369152 t_{14}^{5}+1960220428 t_{14}^{4}-507121440 t_{14}^{3} \\
& +89099433 t_{14}^{2}-9580248 t_{14}+476847=0
\end{aligned}
$$

One singularity configuration in the 2nd Schönflies mode can be obtained by solving Eq. (31), for example $t_{14}=1$. Then the direct kinematics of at least one singularity pose can be computed with $\theta_{11}=0^{\circ}, \theta_{12}=90^{\circ}, \theta_{13}=0^{\circ}, \theta_{14}=90^{\circ}$ and it is shown in Fig. 8 .

The determinant of Jacobian $\operatorname{det}\left(\mathbf{J}_{2}\right)$ also vanishes in two particular conditions, namely when all the actuated joint angles have the same values and when the first links of each limb are pointing inward toward the origin $O$ of the fixed frame $\Sigma_{0}$. In the first condition, when all the actuated joint angles have the same values, the moving platform gains 1-dof self-motion. During the motion, the first links of each limb stay fixed and the moving platform can perform 


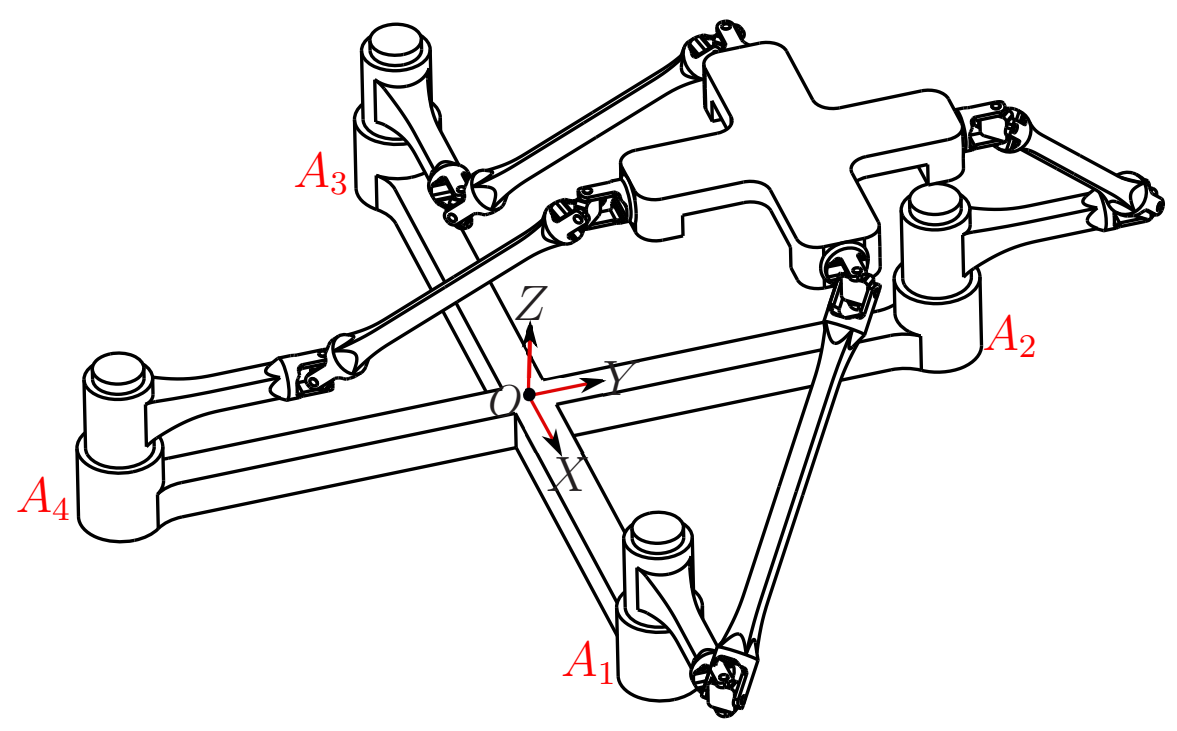

Figure 8: Singularity pose in the 2nd Schönflies mode $\mathcal{K}_{2}$.

a rotational motion. Let us consider the actuated joint angles being $t_{11}=0, t_{12}=0, t_{13}=$ $0, t_{14}=0$ and the 1-dof self-motion is parametrized by $x_{3}$, as shown in Fig. 9.
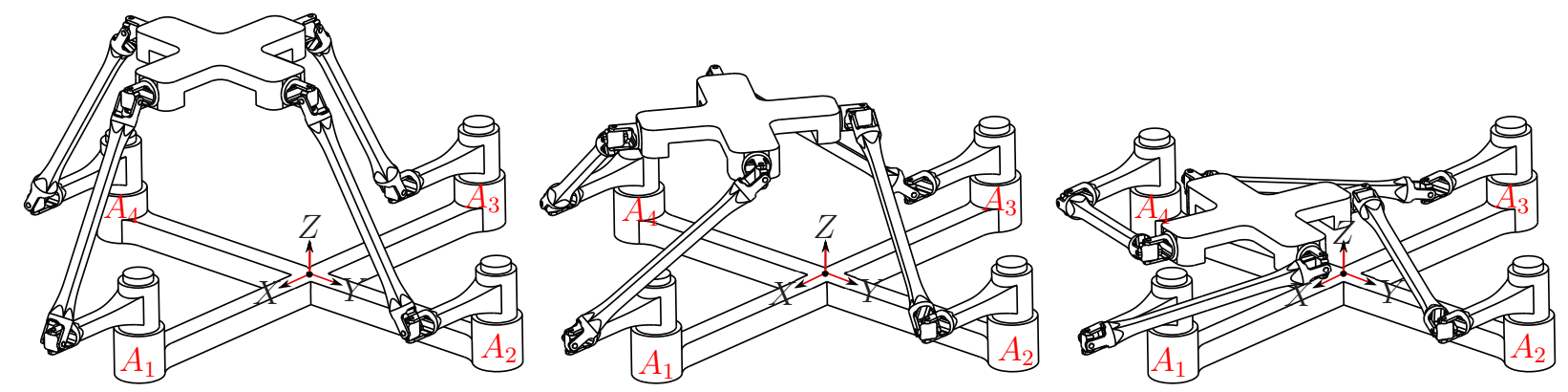

Figure 9: Self-motion when $\theta_{11}=\theta_{12}=\theta_{13}=\theta_{14}=0$ in $\mathcal{K}_{2}$.

In the second condition, the first links of each limb are pointing inward toward the origin $O$ of the fixed frame $\Sigma_{0}$ and the actuated joint angles have the values $\theta_{11}=180^{\circ}, \theta_{12}=-90^{\circ}, \theta_{13}=$ $0^{\circ}, \theta_{14}=90^{\circ}$. Since the tangent half-angle cannot cover $180^{\circ}$, the self-motion is computed with the trigonometric functions. Accordingly, the moving platform gains 3-dof self-motion. During the motion, the first links of each limb stay fixed and the moving platform can perform two translational motions and one rotational motion. Figures 10-12 show the first translational motion, the second translational motion and the rotational motion, respectively. 

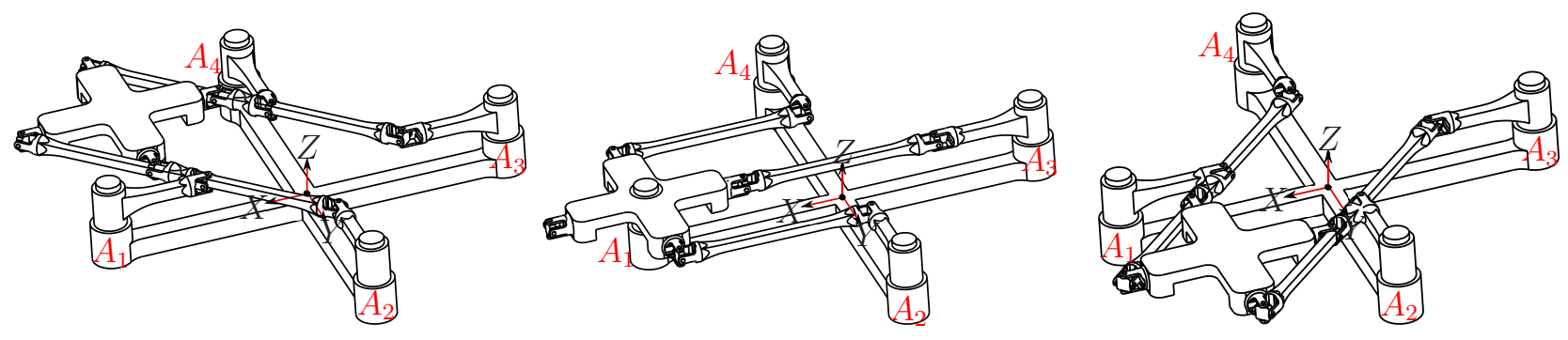

Figure 10: First translation of self-motion in $\mathcal{K}_{2}$.
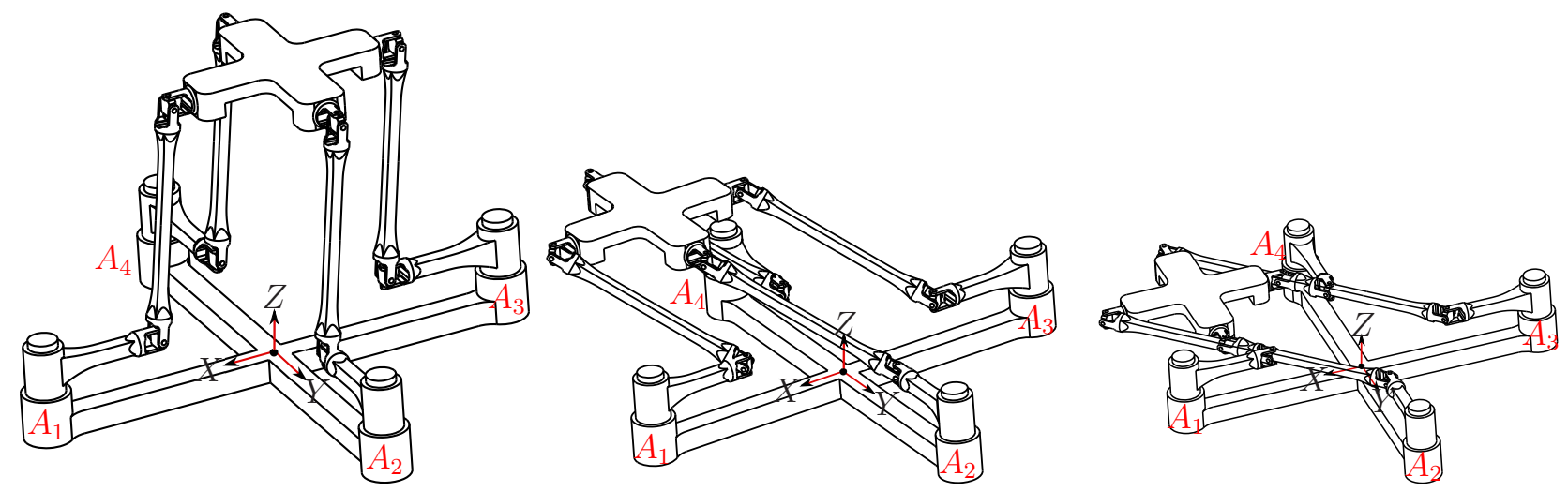

Figure 11: Second translation of self-motion in $\mathcal{K}_{2}$.

\subsection{Self-motions in Third mode $\left(\mathcal{K}_{3}\right)$}

Before computing the self-motion of the 4-RUU PM in the third mode $\mathcal{K}_{3}$, the singularity conditions of the 2-RUU $(I)$ and 2-RUU ${ }_{(I I)} \mathrm{PM}$ in $\mathcal{K}_{3(I)}$ and $\mathcal{K}_{3(I I)}$, respectively, are first discussed. The determinants of the Jacobian matrices are computed in each system $\mathcal{K}_{3(I)}$ and $\mathcal{K}_{3(I I)}$. The determinants of these Jacobian matrices consist of eleven factors that are defined as:

$$
\begin{aligned}
\mathcal{S}_{3(I)} & : \operatorname{det}\left(\mathbf{J}_{3(I)}\right)=\left(t_{21} t_{23}+1\right)\left(-t_{23}+t_{21}\right) x_{0}\left(t_{13}^{2}+1\right)^{3}\left(t_{11}^{2}+1\right)^{3} \ldots=0 \\
\mathcal{S}_{3(I I)}: & \operatorname{det}\left(\mathbf{J}_{3(I I)}\right)=\left(t_{22} t_{24}+1\right)\left(-t_{24}+t_{22}\right) x_{0}\left(t_{14}^{2}+1\right)^{3}\left(t_{12}^{2}+1\right)^{3} \ldots=0
\end{aligned}
$$

It can be seen that the first two factors of $\mathcal{S}_{3(I)}$ and $\mathcal{S}_{3(I I)}$ in Eq. (32) are the necessary conditions for the 2-RUU $U_{(I)}$ and 2-RUU $U_{(I I)} \mathrm{PM}$ to be in the systems $\mathcal{K}_{3(I)}$ and $\mathcal{K}_{3(I I)}$, respectively (as explained in Sections 4.1 and 4.2). They are (1.) $t_{21}=-\frac{1}{t_{23}}\left(\theta_{21}=\pi+\theta_{23}\right)$ and (2.) $t_{21}=t_{23}$ $\left(\theta_{21}=\theta_{23}\right)$ for the 2-RUU $(I)$ PM, and (1.) $t_{22}=-\frac{1}{t_{24}}\left(\theta_{22}=\pi+\theta_{24}\right)$ and (2.) $t_{22}=t_{24}\left(\theta_{22}=\theta_{24}\right)$ for the $2-\mathrm{RUU}_{(I I)} \mathrm{PM}$. It means that each configuration in the systems $\mathcal{K}_{3(I)}$ and $\mathcal{K}_{3(I I)}$ amounts to a self-motion.

To investigate the self-motion in the third mode $\mathcal{K}_{3}$ of the 4-RUU PM, let us recall the first 

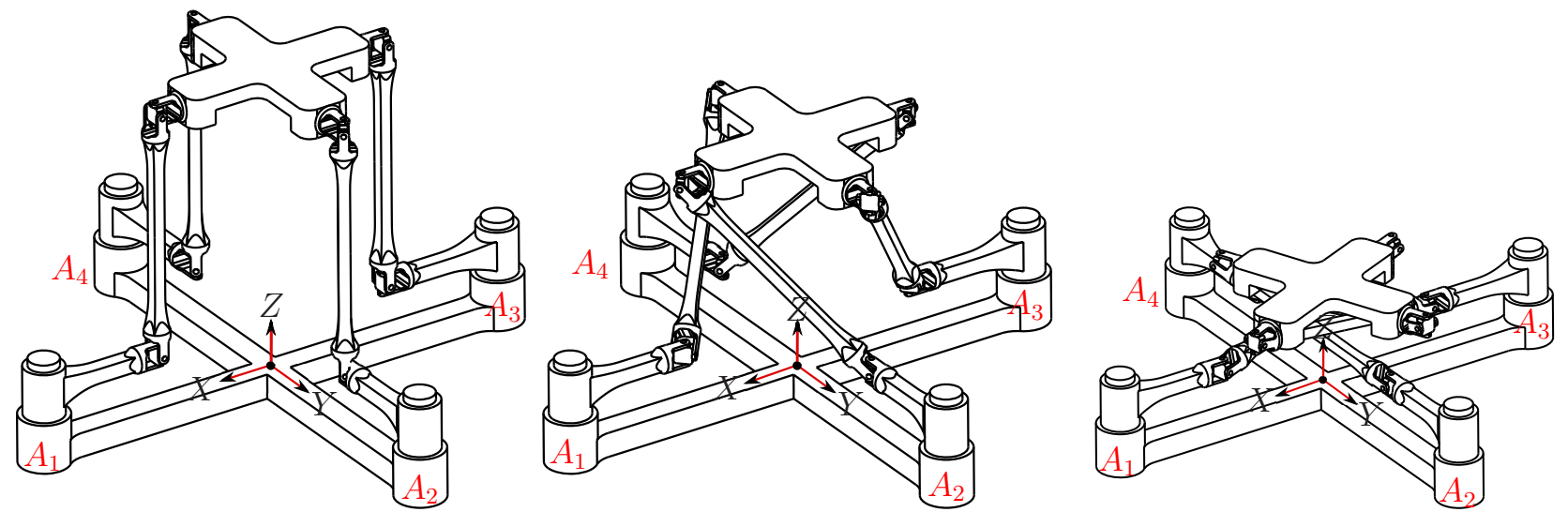

Figure 12: Rotation of self-motion in $\mathcal{K}_{2}$.

factors of ideals $\mathcal{L}_{I}$ and $\mathcal{L}_{I I}$, which have the same mathematical expressions from Section 4.3.1. The variable $x_{1}$ can be solved and it yields two equations in terms of the actuated joint angles only. The first equation is similar to Eq. (28) and the second equation takes the form:

$$
\begin{aligned}
& 3 t_{1}^{2} t_{2}^{2} t_{3}^{2}-3 t_{1}^{2} t_{2}^{2} t_{4}^{2}+3 t_{1}^{2} t_{3}^{2} t_{4}^{2}-3 t_{2}^{2} t_{3}^{2} t_{4}^{2}-t_{1}^{2} t_{2}^{2} t_{3}+t_{1}^{2} t_{2}^{2} t_{4}+2 t_{1}^{2} t_{2} t_{3}^{2}-t_{1}^{2} t_{2} t_{4}^{2}-2 t_{1}^{2} t_{3}^{2} t_{4}+t_{1}^{2} t_{3} t_{4}^{2}-t_{1} t_{2}^{2} t_{3}^{2} \\
& +t_{1} t_{3}^{2} t_{4}^{2}+t_{2}^{2} t_{3}^{2} t_{4}-t_{2} t_{3}^{2} t_{4}^{2}+6 t_{1}^{2} t_{3}^{2}-6 t_{2}^{2} t_{4}^{2}+t_{1}^{2} t_{2}-t_{1}^{2} t_{4}-t_{1} t_{2}^{2}+t_{1} t_{4}^{2}-t_{2}^{2} t_{3}+2 t_{2}^{2} t_{4}+t_{2} t_{3}^{2}-2 t_{2} t_{4}^{2}- \\
& t_{3}^{2} t_{4}+t_{3} t_{4}^{2}+3 t_{1}^{2}-3 t_{2}^{2}+3 t_{3}^{2}-3 t_{4}^{2}=0
\end{aligned}
$$

The variable $x_{2}$ is an independent parameter. As a consequence, when all the corresponding actuated joints are actuated, there is an additional 1-dof rotational motion exhibited by the moving platform. This motion is a self-motion and it is parametrized by the variable $x_{2}$. Two equations in Eq. (28) and Eq. (33) are solved to find the relations among the actuated joint angles in the self-motion, namely:

1. $t_{11}=t_{12}$ and $t_{13}=t_{14} \quad$ or $\quad \theta_{11}=\theta_{12}$ and $\theta_{13}=\theta_{14}$

2. $t_{11}=t_{14}$ and $t_{12}=t_{13} \quad$ or $\quad \theta_{11}=\theta_{14}$ and $\theta_{12}=\theta_{13}$

3. $t_{11}=-\frac{1}{t_{13}}$ and $t_{12}=-\frac{1}{t_{14}} \quad$ or $\quad \theta_{11}=\pi+\theta_{13}$ and $\theta_{12}=\pi+\theta_{14}$

Since two 2-RUU PM are assembled perpendicularly as in the 4-RUU PM, only one example between the self-motion of solutions 1 and 2 is shown. The example of self-motion of solution 2 is shown in Fig. 13 with $\theta_{11}=\theta_{14}=90^{\circ}$ and $\theta_{12}=\theta_{13}=0^{\circ}$. Figure 14 shows the example of self-motion of solution 3 with $\theta_{11}=90^{\circ}, \theta_{12}=0^{\circ}, \theta_{13}=-90^{\circ}, \theta_{14}=180^{\circ}$.

Every configuration in the third modes of the 2-RUU $\mathrm{RI}_{(I)}$ and 2-RUU ${ }_{(I I)} \mathrm{PM}$ amounts to a selfmotion. However, when the 2-RUU $(I)$ and 2-RUU $(I I)$ PM are assembled to obtain the 4-RUU PM, 
every configuration in the third mode $\mathcal{K}_{3}$ of the 4 -RUU PM is not always in self-motion. The self-motion of the third mode $\mathcal{K}_{3}$ occur if and only if the actuated joint angles $\left(t_{11}, t_{12}, t_{13}, t_{14}\right)$ fulfil particular conditions.
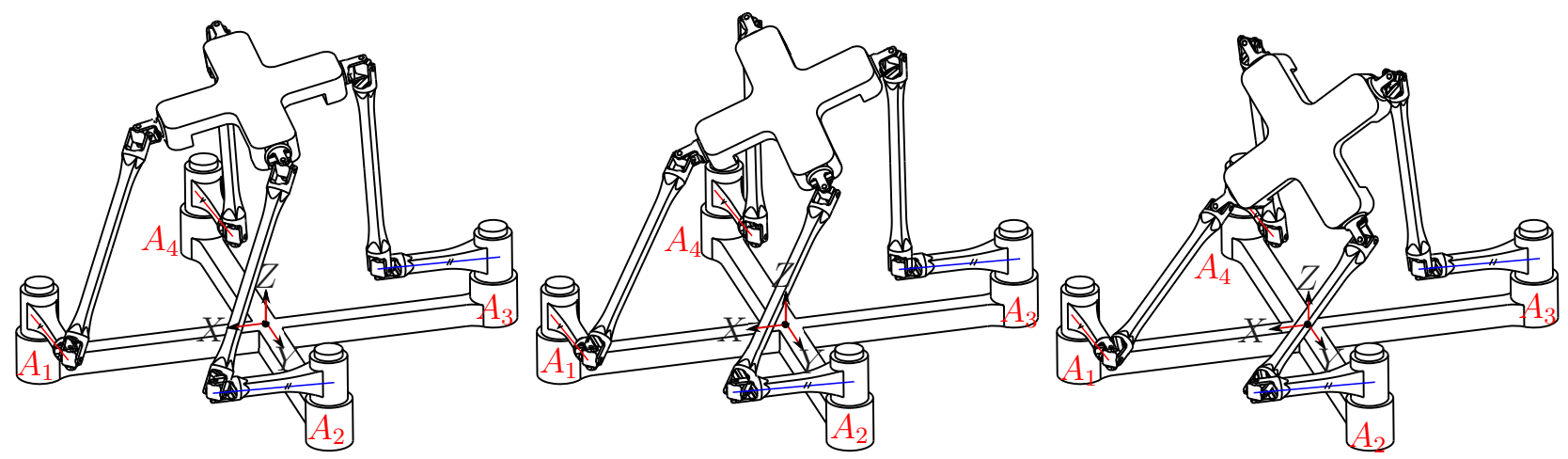

Figure 13: Self-motion when $\theta_{11}=\theta_{14}$ and $\theta_{12}=\theta_{13}$ in $\mathcal{K}_{3}$.
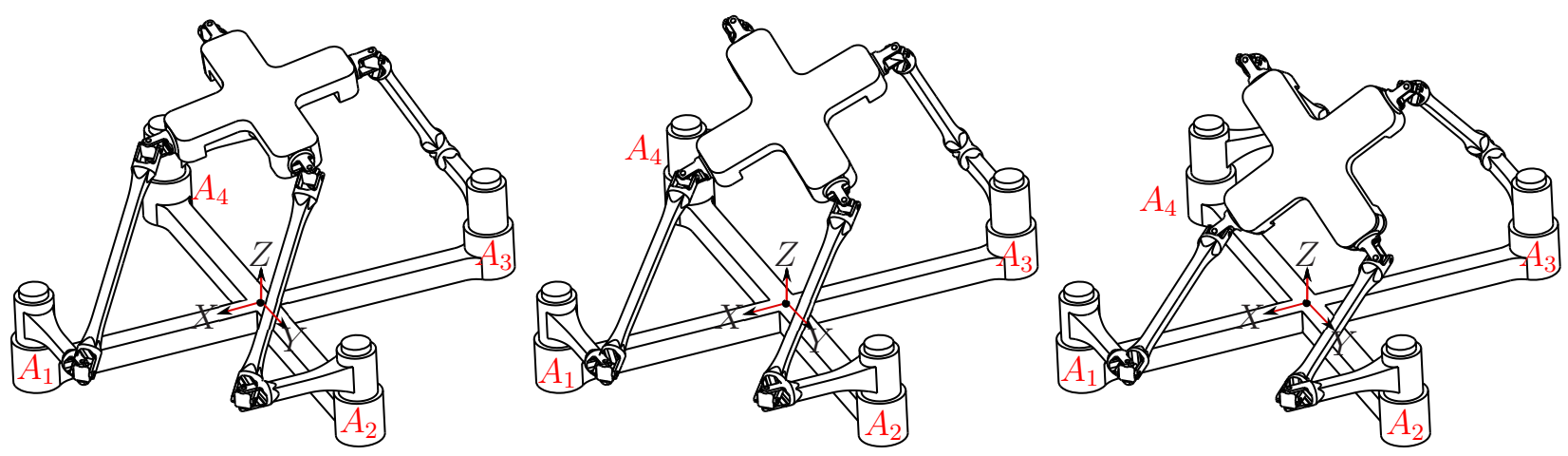

Figure 14: Self-motion when $\theta_{11}=\pi+\theta_{13}$ and $\theta_{12}=\pi+\theta_{14}$ in $\mathcal{K}_{3}$.

\section{Operation mode changing}

There exist common configurations where the mechanism, i.e. the 4-RUU PM, can switch from one operation mode to another operation mode. These configurations are well known as transition configurations. Transition configuration analysis is an important issue in the design process and control of the parallel manipulators with multiple operation modes [2].

However, the 1st Schönflies mode and the 2nd Schönflies mode do not have configurations in common, since the variables $x_{0}, x_{1}, x_{2}, x_{3}$ can never vanish simultaneously. It means that the 4-RUU PM cannot switch from the 1st Schönflies mode to the 2nd Schönflies mode directly. 
To change from the 1st Schönflies mode to the 2nd Schönflies mode, the 4-RUU PM should pass through the third mode, namely system $\mathcal{K}_{3}$. There exist some configurations in which the manipulator can switch from the 1st Schönflies mode to the third mode or vice versa, and these configurations belong to both operation modes. Noticeably, these configurations are also singular configurations since they lie in the intersection of two operation modes.

In the following, the conditions on the actuated joint angles for the 4-RUU PM to change from one operation mode to another are presented. Each pair of ideals $\left\{\mathcal{K}_{i} \cup \mathcal{K}_{j}\right\}$ is analysed and the Study parameters are eliminated to find common solutions.

\subsection{1st Schönflies mode $\left(\mathcal{K}_{1}\right) \longleftrightarrow$ Third mode $\left(\mathcal{K}_{3}\right)$}

To switch from the 1 st Schönflies mode $\left(\mathcal{K}_{1}\right)$ to the third mode $\left(\mathcal{K}_{3}\right)$ or vice versa, one should find the configurations of the 4-RUU PM that fulfil the condition of both operation modes, namely $\left(\mathcal{J}_{1} \cup \mathcal{J}_{3}\right)$. Then all Study parameters are eliminated to find an equation in terms of the actuated joint angles $t_{11}, t_{12}, t_{13}, t_{14}$, written as:

$$
\begin{aligned}
& 9 t_{11}^{12} t_{12}^{12} t_{13}^{12} t_{14}^{6}-54 t_{11}^{12} t_{12}^{12} t_{13}^{11} t_{14}^{7}+135 t_{11}^{12} t_{12}^{12} t_{13}^{10} t_{14}^{8}+54 t_{11}^{12} t_{12}^{11} t_{13}^{12} t_{14}^{7}-270 t_{11}^{12} t_{12}^{11} t_{13}^{11} t_{14}^{8}+135 t_{11}^{12} t_{12}^{10} t_{13}^{12} t_{14}^{8} \\
& -54 t_{11}^{11} t_{12}^{12} t_{13}^{12} t_{14}^{7}+270 t_{11}^{11} t_{12}^{12} t_{13}^{11} t_{14}^{8}-270 t_{11}^{11} t_{12}^{11} t_{13}^{12} t_{14}^{8}+135 t_{11}^{10} t_{12}^{12} t_{13}^{12} t_{14}^{8}-180 t_{11}^{12} t_{12}^{12} t_{13}^{9} t_{14}^{9} \ldots=0
\end{aligned}
$$

In this transition configurations, the moving platform is twisted about an axis parallel to $X Y$-plane of $\Sigma_{0}$ by 180 degrees and the actuated joint angles fulfil Eq. (34). The three conditions of the self-motions (1.) $t_{11}=t_{12}$ and $t_{13}=t_{14}$, (2.) $t_{11}=-1 / t_{13}$ and $t_{12}=-1 / t_{14}$, and (3.) $t_{11}=t_{14}$ and $t_{12}=t_{13}$ given in Section 5, are contained in Eq. (34). It shows that the moving platform is in a transition configuration of the 1 st Schönflies mode $\mathcal{K}_{1}$ and the third mode $\mathcal{K}_{3}$ that amounts to a self-motion.

\subsection{2nd Schönflies mode $\left(\mathcal{K}_{2}\right) \longleftrightarrow$ Third mode $\left(\mathcal{K}_{3}\right)$}

To switch from the 2 nd Schönflies mode $\left(\mathcal{K}_{2}\right)$ to the third mode $\left(\mathcal{K}_{3}\right)$ or vice versa, one should find the configurations of the 4-RUU PM that fulfil the condition of both operation modes, namely $\left(\mathcal{J}_{2} \cup \mathcal{J}_{3}\right)$. Then all Study parameters are eliminated to find an equation in terms of the actuated joint angles $t_{11}, t_{12}, t_{13}, t_{14}$, written as:

$$
\begin{aligned}
& 9 t_{11}^{12} t_{12}^{12} t_{13}^{12} t_{14}^{6}-54 t_{11}^{12} t_{12}^{12} t_{13}^{11} t_{14}^{7}+135 t_{11}^{12} t_{12}^{12} t_{13}^{10} t_{14}^{8}-180 t_{11}^{12} t_{12}^{12} t_{13}^{9} t_{14}^{9}+135 t_{11}^{12} t_{12}^{12} t_{13}^{8} t_{14}^{10}-54 t_{11}^{12} t_{12}^{12} t_{13}^{7} t_{14}^{11} \\
& +9 t_{11}^{12} t_{12}^{12} t_{13}^{6} t_{14}^{12}+54 t_{11}^{12} t_{12}^{11} t_{13}^{12} t_{14}^{7}-270 t_{11}^{12} t_{12}^{11} t_{13}^{11} t_{14}^{8}+540 t_{11}^{12} t_{12}^{11} t_{13}^{10} t_{14}^{9}-540 t_{11}^{12} t_{12}^{11} t_{13}^{9} t_{14}^{10}+\ldots=0
\end{aligned}
$$


The moving platform of the 4-RUU PM is in a transition configuration between $\mathcal{K}_{2}$ and $\mathcal{K}_{3}$ when the moving platform is parallel to the base and the actuated joint angles fulfil Eq. (35). The three conditions of the self-motions (1.) $t_{11}=t_{12}$ and $t_{13}=t_{14}$, (2.) $t_{11}=-1 / t_{13}$ and $t_{12}=-1 / t_{14}$, and (3.) $t_{11}=t_{14}$ and $t_{12}=t_{13}$ given in Section 5, are contained in Eq. (35). It means that the moving platform is in a transition configuration of the 2nd Schönflies mode $\mathcal{K}_{2}$ and the third mode $\mathcal{K}_{3}$ that amounts to a self-motion. As a consequence, the transition between systems $\mathcal{K}_{1}$ and $\mathcal{K}_{2}$ occurs through the third system $\mathcal{K}_{3}$, which is lower dimension operation mode and amounts to a self-motion. The transition from $\mathcal{K}_{2}$ to $\mathcal{K}_{1}$ through the third mode $\mathcal{K}_{3}$ with the condition of the actuated joint angles $t_{11}=t_{12}$ and $t_{13}=t_{14}$, is shown in Fig. 15(a)-15(f).

\section{Conclusions}

In this paper, the method of algebraic geometry was applied to characterize the type of operation modes of the 4-RUU PM. The 4-RUU PM is initially decomposed into two 2-RUU PM. The constraint equations corresponding to two 2-RUU PM are derived and the primary decomposition is computed. It reveals that the 2-RUU PM have three 4-dof operation modes. However, when they are assembled to be the 4-RUU PM, its operation modes are composed of two 4-dof Schönflies modes and one 2-dof operation mode.

The singularity conditions were computed and represented in the joint space, i.e., the actuated joint angles $\left(t_{11}, t_{12}, t_{13}, t_{14}\right)$. It turns out that every configuration in the 4-dof third modes of both 2-RUU PM, is always in singularity and it amounts to a self-motion. However, every configuration in the 2-dof third mode of the 4-RUU PM is not always in singularity, i.e., self-motion. The self-motion in this operation mode occurs if the actuated joint angles fulfil some particular conditions, namely (1.) $t_{11}=t_{12}$ and $t_{13}=t_{14}$, (2.) $t_{11}=-1 / t_{13}$ and $t_{12}=-1 / t_{14}$, and (3.) $t_{11}=t_{14}$ and $t_{12}=t_{13}$. The 4-RUU PM is able to switch from the 1st Schönflies mode to the 2nd Schönflies mode by passing through the third mode, which contains self-motion.

\section{References}

[1] Zlatanov, D., Bonev, I.A., and Gosselin, C.M. (2002). "Constraint Singularities as CSpace Singularities,", The 8th International Symposium on Advances in Robot Kinematics, Caldes de Malavella, Spain, June 24-28, pp. 183-192. 


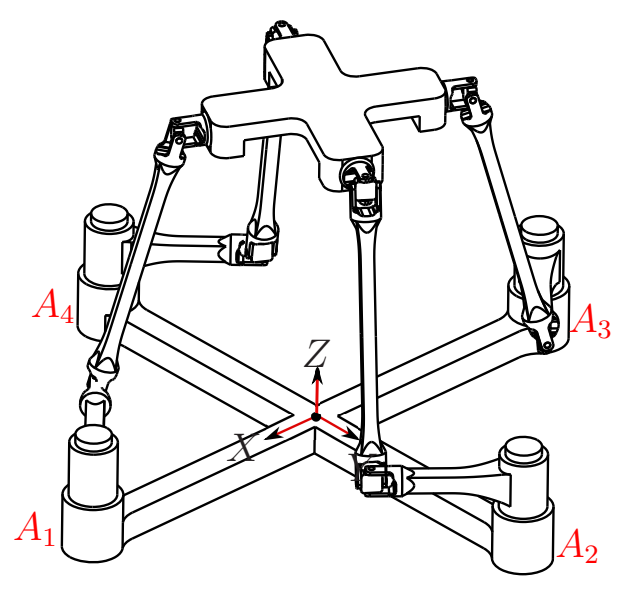

(a) 2nd Schönflies mode

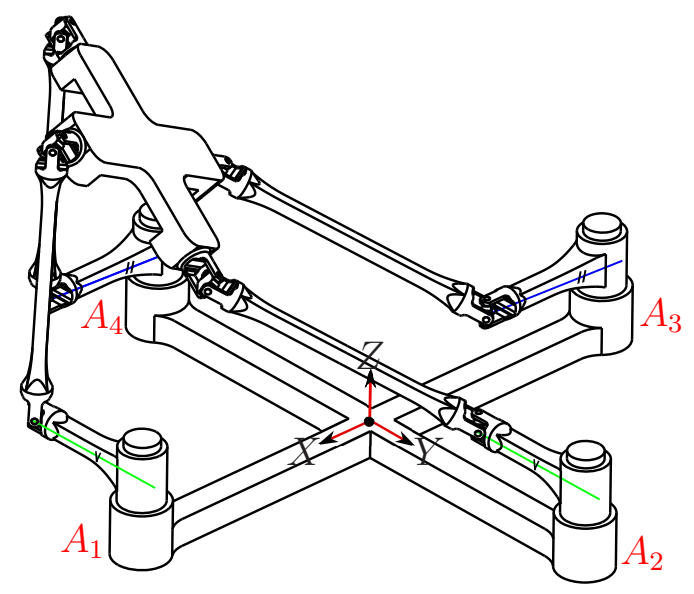

(c) Third mode

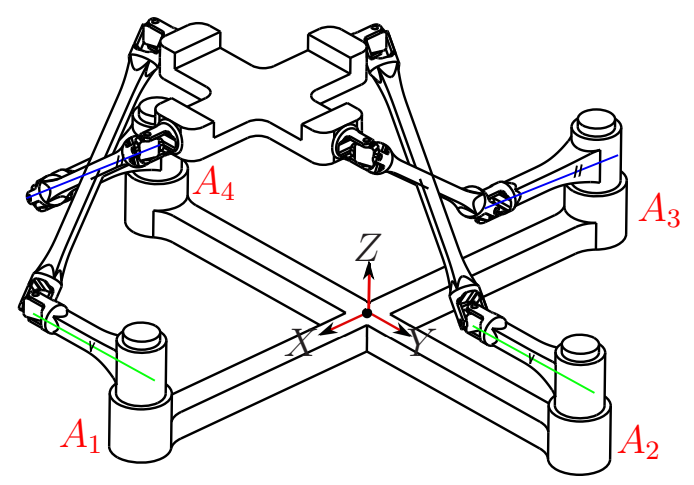

(e) Transition configuration

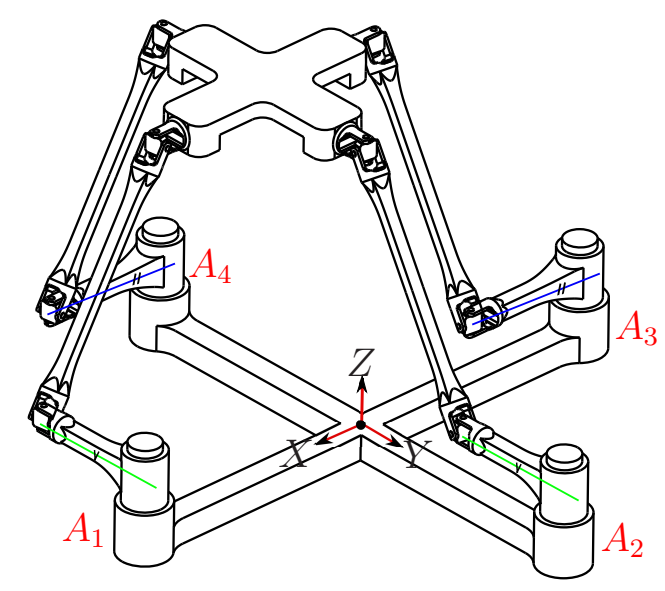

(b) Transition configuration

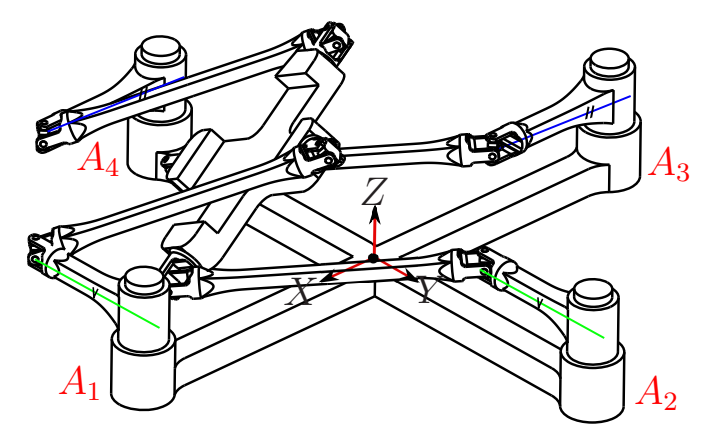

(d) Third mode

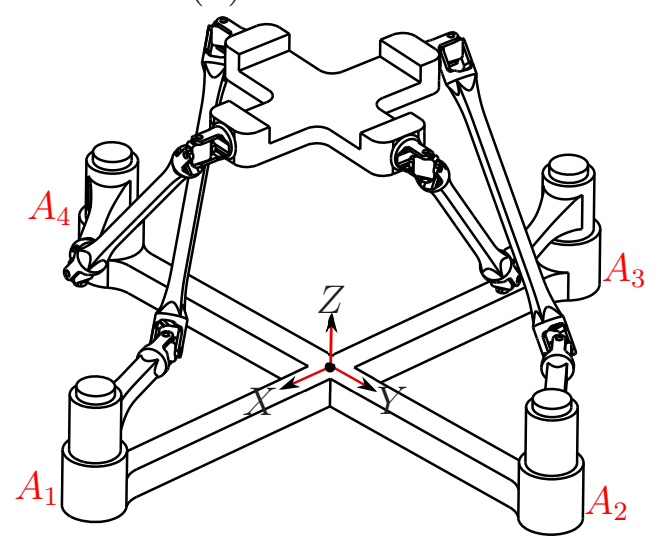

(f) 1 st Schönflies mode

Figure 15: Transition from the 2nd Schönflies mode to the 1st Schönflies mode via the third mode with $\theta_{11}=\theta_{12}$ and $\theta_{13}=\theta_{14}$. 
[2] Kong, X. (2014). "Reconfiguration Analysis of a 3-DOF Parallel Mechanism Using Euler Parameter Quaternions and Algebraic Geometry Method", Mechanism and Machine Theory, 74, pp. 188-201.

[3] Walter, D.R., Husty, M.L., and Pfurner, M. (2009). "A Complete Kinematic Analysis of the SNU 3-UPU Parallel Robot", Contemporary Mathematics, 496, pp. 331-346.

[4] Walter, D.R. and Husty, M.L. (2011). "Kinematic Analysis of the TSAI 3-UPU Parallel Manipulator Using Algebraic Methods", The 13th IFToMM World Congress in Mechanism and Machine Science, Guanajuato, Mexico, June 19-25, pp. 1-10.

[5] Tsai, L.-W. (1996). "Kinematics of a 3-DOF Platform with Three Extensible Limbs", In: Lenarčič, J and V. Parenti-Castelli, V, Recent Advances in Robot Kinematics, Kluwer Academic Publisher, pp. 401-410.

[6] Schadlbauer, J., Walter, D.R., and Husty, M. (2014). "The 3-RPS Parallel Manipulator from an Algebraic Viewpoint", Mechanism and Machine Theory, 75, pp. 161-176.

[7] Hunt, K.H. (1983). "Structural Kinematics of In-Parallel-Actuated Robot-Arms", ASME Journal of Mechanisms, Transmissions, and Automation in Design, 105(67), pp. 705-712.

[8] Schadlbauer, J., Nurahmi, L., Husty, M., Wenger, P., and Caro, S. (2013). "Operation Modes in Lower-Mobility Parallel Manipulators", In: Kecskeméthy, A and Geu Flores, F, Interdisciplinary Applications of Kinematics, 26, Springer, pp. 1-9.

[9] Schadlbauer, J., Husty, M., Caro, S., and Wenger, P. (2013). "Self-Motions of 3-RPS Manipulators", Frontiers of Mechanical Engineering, 8(10), pp. 62-69.

[10] Huang, Z and Fang, Y. (1995). "Motion Characteristics and Rotational Axis Analysis of Three DOF Parallel Robot Mechanisms", IEEE International Conference on Systems, Man, and Cybernetics, pp. 67-71.

[11] Nurahmi, L., Schadlbauer, J., Husty, M., Caro, S., and Wenger, P. (2015). "Kinematic Analysis of the 3-RPS Cube Parallel Manipulator", ASME Journal of Mechanisms and Robotics, 7(1), pp. 011008-1-011008-11.

[12] Nurahmi, L., Schadlbauer, J., Husty, M., Wenger, P., and Caro, S. (2015). "Motion Capability of the 3-RPS Cube Parallel Manipulator", In: Lenarčič, J and Khatib, O, Advances in Robot Kinematics, Springer, pp. 527-535. 
Nurahmi, Caro, Wenger, Schadlbauer, and Husty, submitted to Mech. and Mach. Theory 30

[13] Kong, X., Gosselin, C.M., and Richard, P-L. (2007). "Type Synthesis of Parallel Mechanisms with Multiple Operation Modes", ASME Journal of Mechanical Design, 129(6), pp. 595-601.

[14] Kong, X. (2012). "Type Synthesis of Variable Degrees-of-Freedom Parallel Manipulators with Both Planar and 3T1R Operation Modes", Proceedings of the ASME 2012 International Design Engineering Technical Conference and Information in Engineering Conference, Chicago, USA, August 12-15.

[15] He, X., Kong, X., Chablat, D., Caro, S., and Hao, G. (2014). "Kinematic Analysis of a Single-Loop Reconfigurable 7R Mechanism with Multiple Operation Modes", Robotica, pp. 1-18.

[16] Kong, X. and Pfurner, M. (2015). "Type Synthesis and Reconfiguration Analysis of a Class of Variable-DOF Single-Loop Mechanisms", Mechanism and Machine Theory, 85, pp. 116-128.

[17] Fanghella, P., Galletti, C., and Giannotti, E. (2006). "Parallel Robots that Change Their Group of Motion", In: Lenarčič, J and Roth, B, Advances in Robot Kinematics, Springer Netherlands, pp. 49-56.

[18] Refaat, S., Hervé, J., Nahavandi, S., and Trinh, H. (2006). "Two-Mode Overconstrained Three DOFs Rotational-Translational Linear-Motor-Based Parallel-Kinematics Mechanism for Machine Tool Applications", Robotica, 25, pp. 461-466.

[19] Gogu, G. (2011). "Maximally Regular T2R1-Type Parallel Manipulators with Bifurcated Spatial Motion", ASME Journal of Mechanisms and Robotics, 3(1), pp. 011010.

[20] Gan, D., Dai, J.S., Liao, Q. (2007). "Mobility Change in Two Types of Metamorphic Parallel Mechanisms", ASME Journal of Mechanisms and Robotics, 1(4), pp. 041007.

[21] Gan, D., Dai, J.S., Dias, J., and Seneviratne, L. (2013). "Unified Kinematics and Singularity Analysis of a Metamorphic Parallel Mechanism with Bifurcated Motion". ASME Journal of Mechanisms and Robotics, 5, pp. 031004-1-031004-11.

[22] Dai, J.S. and Rees Jones, J. (1999). "Mobility in Metamorphic Mechanisms of Foldable/Erectable Kinds". ASME Journal of Mechanical Design, 121(1), pp. 375-382.

[23] Wohlhart, K. (1996). "Kinematotropic linkages". In: Lenarčič, J and Parenti-Castelli, V, Advances in robot Kinematics, Kluwer, Dordrecht, pp. 359-368. 
Nurahmi, Caro, Wenger, Schadlbauer, and Husty, submitted to Mech. and Mach. Theory 31

[24] Li, Q. and Hervé, J. (2009). "Parallel Mechanisms with Bifurcation of Schoenflies Motion", IEEE Transactions on Robotics, 25(1), pp. 158-164.

[25] Lee, C-C. and Hervé, J. (2011). "Isoconstrained Parallel Generators of Schoenflies Motion", ASME Journal of Mechanisms and Robotics, 3(2), pp. 021006.

[26] Kong, X. and Gosselin, C.M. (2007). "Type Synthesis of Parallel Mechanisms", Springer, Heidelberg, 33, pp. 141-157.

[27] Masouleh, M.T., Walter, D.R., Husty, M., and Gosselin, C.M. (2011). "Solving the Forward Kinematic Problem of 4-DOF Parallel Mechanisms (3T1R) with Identical Limb Structures and Revolute Actuators Using the Linear Implicitization Algorithm", Proceedings of the ASME 2012 International Design Engineering Technical Conference and Information in Engineering Conference, Washington, USA, August 28-31.

[28] Amine, S., Masouleh, M.T., Caro, S., Wenger, P., and Gosselin, C.M. (2011). "Singu-

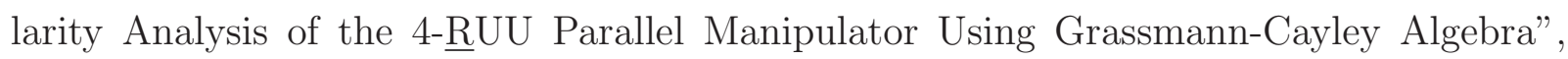
Transactions of the Canadian Society for Mechanical Engineering, 35(4), pp. 515-528.

[29] Amine, S., Masouleh, M.T., Caro, S., and Wenger, P. (2012). "Singularity Conditions of 3T1R Parallel Manipulators with Identical Limb Structures", ASME Journal of Mechanisms and Robotics, 4, pp. 011011-1-011011-11.

[30] Husty, M., Pfurner, M., Shröcker, H-P., and Brunnthaler, K. (2007). "Algebraic Methods in Mechanism Analysis and Synthesis", Robotica, 25(6), pp. 661-675.

[31] Nurahmi, L., Caro, S., and Wenger, P. (2015). "Operation Modes and Self-motions of a 2RUU Parallel Manipulator", In: Bai, S and Ceccarelli, M, Recent Advances in Mechanism Design for Robotics, 33, Springer, pp. 417-426. 